Geometry \& Topology

Volume 9 (2005) 1775-1834

Published: 25 September 2005

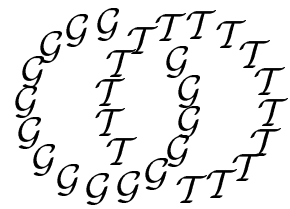

\title{
Squeezing in Floer theory and refined Hofer-Zehnder capacities of sets near symplectic submanifolds
}

\author{
ELY KeRMAN \\ Mathematics, University of Illinois at Urbana-Champaign \\ Urbana, IL 61801, USA \\ and \\ Institute of Science, Walailak University \\ Nakhon Si Thammarat, 80160, Thailand \\ Email: ekerman@math.uiuc.edu \\ URL: http://www.math.uiuc.edu/ ekerman/
}

\begin{abstract}
We use Floer homology to study the Hofer-Zehnder capacity of neighborhoods near a closed symplectic submanifold $M$ of a geometrically bounded and symplectically aspherical ambient manifold. We prove that, when the unit normal bundle of $M$ is homologically trivial in degree $\operatorname{dim}(M)$ (for example, if $\operatorname{codim}(M)>\operatorname{dim}(M))$, a refined version of the Hofer-Zehnder capacity is finite for all open sets close enough to $M$. We compute this capacity for certain tubular neighborhoods of $M$ by using a squeezing argument in which the algebraic framework of Floer theory is used to detect nontrivial periodic orbits [18, 19, 26. As an application, we partially recover some existence results of Arnold [1] for Hamiltonian flows which describe a charged particle moving in a nondegenerate magnetic field on a torus. Following [43, we also relate our refined capacity to the study of Hamiltonian paths with minimal Hofer length.
\end{abstract}

AMS Classification numbers Primary: 53D40

Secondary: $37 \mathrm{~J} 45$

Keywords: Hofer-Zehnder capacity, symplectic submanifold, Floer homology

Proposed: Leonid Polterovich

Received: 22 March 2005

Seconded: Yasha Eliashberg, Eleny Ionel

Revised: 11 September 2005

(c) Geometry ${ }^{\mathcal{S}} \mathcal{T}$ Topology Publications 


\section{Introduction and results}

The Hofer-Zehnder capacity of an open subset of a symplectic manifold, is a measure of the size of the set in terms of the periodic orbits of the autonomous Hamiltonian flows it supports. This symplectic invariant was introduced by Hofer and Zehnder in 38, where the authors also compute its value for the standard symplectic cylinder $Z^{2 l}(R)=B^{2}(R) \times \mathbb{R}^{2 l-2}$ in $\left(\mathbb{R}^{2 l}, \Omega_{2 l}\right)$. In particular, they prove that

$$
c_{\mathrm{HZ}}\left(Z^{2 l}(R)\right)=\pi R^{2} .
$$

This remarkable result illustrates the symplectic nature of the Hofer-Zehnder capacity by distinguishing it from the volume. Moreover, it yields an alternative proof of Gromov's famous nonsqueezing theorem and hence it serves as an important link between the existence problem for periodic orbits of Hamiltonian flows and symplectic rigidity phenomena 39].

Viewing the symplectic cylinder $Z^{2 l}(R)$ as a tubular neighborhood of the symplectic submanifold $\mathbb{R}^{2 l-2} \subset \mathbb{R}^{2 l}$, equation (11) naturally leads one to the following question which motivates this work.

Let $M$ be a closed symplectic submanifold of a symplectic manifold $(W, \Omega)$, and let $U_{R}$ be a symplectic tubular neighborhood of $M$ with (sufficiently small) radius $R$. Is the Hofer-Zehnder capacity of $U_{R}$ equal to $\pi R^{2}$ ?

Of course, to make this precise one must specify what is meant by a symplectic tubular neighborhood of radius $R$. These are neighborhoods of $M$ on which the symplectic form has a standard normal form. They are defined below in Section 3 .

It follows from Weinstein's Symplectic Neighborhood Theorem that any symplectic invariant of $U_{R}$ is completely determined by three factors; the radius $R$, the restriction of $\Omega$ to $M$, and the isomorphism class of the normal bundle of $M$ in $W$. The real question then is whether the Hofer-Zehnder capacity is independent of the last two factors. It is interesting to note that an affirmative answer in either case would further distinguish the Hofer-Zehnder capacity from the volume. In particular, Hermann Weyl's famous formulas for the volumes of tubes [30, 66], demonstrate that the volume of a tubular neighborhood of a submanifold depends, in general, on both the volume of the submanifold as well as the class of its normal bundle.

The question above is also relevant to several active areas of research in Hamiltonian dynamics and symplectic topology. In the study of the Hamiltonian flows which describe the classical motion of a charged particle in an electro-magnetic 
field, an affirmative answer to this question immediately implies the existence of periodic orbits on almost all low energy levels (see, for example, [12, 26, 59] and Section 2.2 below). Following the work of Lalonde and McDuff from [44, this question is also of great importance in the study of Hamiltonian paths which minimize the Hofer length [53. As well, Biran's decomposition theorem from [5] shows that a closed Kähler manifolds can, in some suitable sense, be "filledup" by a symplectic tubular neighborhood. Hence, an affirmative answer to the question above would have many implications for the symplectic topology of closed Kähler manifolds.

The first results concerning this question were obtained for trivial symplectic tubular neighborhoods of the form $M \times B^{2}(R)$, where $M$ is closed and the product is given the obvious split symplectic form. These began with the following theorem.

Theorem 1.1 (Floer-Hofer-Viterbo [20]) If $(M, \omega)$ is weakly exact, that is, if $\omega$ vanishes on $\pi_{2}(M)$, then $c_{\mathrm{HZ}}\left(M \times B^{2}(R)\right)=\pi R^{2}$.

This was later extended to the case when $(M, \omega)$ is rational by Hofer and Viterbo in [36. In the context of studying length minimizing Hamiltonian paths, McDuff and Slimowitz also proved more general results for slightly different capacities in [53]. The result for general symplectic manifolds $(M, \omega)$ was proved recently by G. Lu in [49] using the theory of Gromov-Witten invariants and, in particular, Liu and Tian's construction of an equivariant virtual moduli cycle from [4].

In this paper, we prove that a refined Hofer-Zehnder capacity of a symplectic tubular neighborhood $U_{R}$ is equal to $\pi R^{2}$ for all small $R>0$, provided that the ambient manifold $(W, \Omega)$ is geometrically bounded and symplectically aspherical, and the homology of the unit normal bundle of $M$ splits in degree $2 m=\operatorname{dim}(M)$. To state the result precisely, we must first recall the definition of the Hofer-Zehnder capacity and introduce the refinements considered here.

Remark 1.2 Several other recent works [5, 12, 26, 50, 59] have also been devoted to this question. A comparison of our results and methods to those from [5, 12, 26, 50, 59] is contained in Sections 1.3 and 1.4 below.

\section{$1.1 \quad$ Hofer-Zehnder capacities}

On a symplectic manifold $(W, \Omega)$ every Hamiltonian $H: W \rightarrow \mathbb{R}$ determines a unique Hamiltonian vector field $X_{H}$ via the equation

$$
\Omega\left(X_{H}, \cdot\right)=-d H(\cdot) .
$$


If $d H$ is compactly supported, then the flow of $X_{H}$ is defined for all time (we will also refer to this as the Hamiltonian flow of $H$ ). We denote the flow for time $t$ by $\phi_{H}^{t}$.

Let $U$ be a nonempty open subset of $W$. The set of test Hamiltonians on $U$, $\mathcal{H}_{\text {test }}(U)$, is defined to be the set of smooth nonnegative functions $H: W \rightarrow$ $[0, \infty)$ with the following properties:

- $H$ has compact support contained in $U$.

- The set on which $H$ takes the value zero has nonempty interior.

- The set on which $H$ attains its maximum value, $\max (H)$, has nonempty interior.

A test Hamiltonian $H$ is said to be admissible if:

(*) The Hamiltonian flow of $H$ has no nonconstant periodic orbits with period less than or equal to one.

The Hofer-Zehnder capacity of $U$ in $(W, \Omega)$ is then defined to be

$$
c_{\mathrm{HZ}}(U)=\sup \left\{\max (H) \mid H \in \mathcal{H}_{\text {test }}(U), H \text { satisfies }(*)\right\} .
$$

By changing the admissibility criterion and/or the set of test Hamiltonians one obtains different Hofer-Zehnder capacities, [26, 48, 50, 60]. Here we consider a weaker admissibility criterion which is defined using the action functional on $\mathcal{L}$, the space of smooth contractible loops in $W$. For this reason, we will assume that $(W, \Omega)$ is weakly exact, ie $\left.\Omega\right|_{\pi_{2}(W)}=0$. Under this assumption, each smooth map $x: S^{1} \rightarrow W$ has a well-defined symplectic area

$$
\mathcal{A}(x)=\int_{D^{2}} v^{*} \omega
$$

where $v$ is any smooth map from the disc $D^{2}$ to $W$ which satisfies $\left.v\right|_{\partial D^{2}}=x$. For a fixed $H \in \mathcal{H}_{\text {test }}(U)$, the action of $x \in \mathcal{L}$ is then defined by

$$
\mathcal{A}_{H}(x)=\int_{0}^{1} H(x(t)) d t-\mathcal{A}(x) .
$$

The critical points of the action functional $\mathcal{A}_{H}: \mathcal{L} \rightarrow \mathbb{R}$ are precisely the contractible periodic orbits of $H$ with period equal to one. A simple, but crucial, observation is that the $\mathcal{A}_{H}$-value of any constant periodic orbit of $H$ must be less than or equal to $\max (H)$. This leads us to the following admissibility criteria for Hamiltonians on weakly exact manifolds. 
$(*)^{1, \kappa}$ The Hamiltonian flow of $H$ has no nonconstant contractible periodic orbits with period equal to one and action in the interval

$$
(\max (H), \max (H)+\kappa)
$$

for $\kappa>0$.

The corresponding capacity of an open subset $U \subset W$ is then defined by

$$
c_{\mathrm{HZ}}^{1, \kappa}(U)=\sup \left\{\max (H) \mid H \in \mathcal{H}_{\text {test }}(U), H \text { satisfies }(*)^{1, \kappa}\right\} .
$$

This refined capacity contains the maximum amount of information yielded by the squeezing techniques we employ here. The effort invested in retaining this information is repaid by the fact that the almost existence theorem for this capacity includes a bound for the symplectic area of the periodic orbits, (Theorem 2.2). Similar area bounds were obtained by Hofer and Zehnder in their original work [37] on dense existence results in $\left(\mathbb{R}^{2 l}, \Omega_{2 l}\right)$. These bounds, as well as some applications, are described in Section 2.

The invariant $c_{\mathrm{HZ}}^{1, \kappa}$ has the usual properties of a symplectic capacity:

(1) Invariance If $\phi$ is a symplectomorphism of $(W, \Omega)$, then

$$
c_{\mathrm{HZ}}^{1, \kappa}(U)=c_{\mathrm{HZ}}^{1, \kappa}(\phi(U)) .
$$

(2) Monotonicity If $U \subset V$, then $c_{\mathrm{HZ}}^{1, \kappa}(U) \leq c_{\mathrm{HZ}}^{1, \kappa}(V)$.

(3) Homogeneity Let the notation $c_{\mathrm{HZ}}^{1, \kappa}(U, \Omega)$ reflect the dependence of the capacity on the symplectic form. Then for any constant $\alpha>0$

$$
c_{\mathrm{HZ}}^{1, \kappa}(U, \alpha \Omega)=|\alpha| c_{\mathrm{HZ}}^{1, \kappa /|\alpha|}(U, \Omega) .
$$

(4) Normalization If $(W, \Omega)=\left(\mathbb{R}^{2 l}, \Omega_{2 l}\right)$, then

$$
c_{\mathrm{HZ}}^{1,2 \pi R^{2}}\left(B^{2 l}(R)\right)=\pi R^{2}=c_{\mathrm{HZ}}^{1,2 \pi R^{2}}\left(Z^{2 l}(R)\right) .
$$

The first three of these properties follow easily from the definition [39]. The normalization condition, in particular the fact that

$$
c_{\mathrm{HZ}}^{1,2 \pi R^{2}}\left(Z^{2 l}(R)\right)=\pi R^{2},
$$

can be proved using the methods developed here.

We also note that for all $\kappa^{\prime}>\kappa>0$ we have

$$
c_{\mathrm{HZ}}^{1, \kappa}(U) \geq c_{\mathrm{HZ}}^{1, \kappa^{\prime}} \geq c_{\mathrm{HZ}}(U) .
$$

Moreover, since our capacity only detects contractible periodic orbits, there are sets $U$ with $c_{\mathrm{HZ}}^{1, \kappa}(U)=\infty$ and $c_{\mathrm{HZ}}(U)<\infty$. (For example, let $U$ be a neighborhood of a noncontractible loop in $\mathbb{T}^{2}$.) 


\subsection{The main result}

For the remainder of the paper our ambient symplectic manifold will be $(W, \Omega)$, and $M \subset W$ will be a closed symplectic submanifold of dimension $2 m$ and codimension $2 n$. The restriction of $\Omega$ to $M$ will be denoted by $\omega$.

We will assume that $(W, \Omega)$ is symplectically aspherical. That is, $\left.\Omega\right|_{\pi_{2}(W)}=0$ and $\left.c_{1}\right|_{\pi_{2}(W)}=0$, where the forms $\Omega$ and $c_{1}$ act on $\pi_{2}(W)$ by integration over (piecewise) smooth representatives.

We also assume that $(W, \Omega)$ is geometrically bounded, which means that $W$ admits an almost complex structure $J$ and a complete Riemannian metric $g$ for which

- there are positive constants $c_{1}$ and $c_{2}$ such that

$$
\Omega(X, J X) \geq c_{1}\|X\|^{2} \text { and }|\Omega(X, Y)| \leq c_{2}\|X\|\|Y\|
$$

for all $X, Y \in T W$,

- the sectional curvature of $(W, g)$ is bounded from above and the injectivity radius of $(W, g)$ is bounded away from zero.

This assumption is trivial when $W$ is compact. For noncompact manifolds it implies the required $C^{0}$-bounds for the Floer moduli spaces.

Finally, let $E \rightarrow M$ be a normal bundle of $M$ in $W$, and let $S(E)$ be the corresponding unit normal bundle with respect to some fibrewise metric on $E$. We say that $S(E)$ is homologically trivial in degree $2 m$, if the homology of $S(E)$ (with coefficients in $\mathbb{Z}_{2}$ ) splits in degree $2 m$, ie

$$
H_{2 m}\left(S(E), \mathbb{Z}_{2}\right)=H_{2 m}\left(M, \mathbb{Z}_{2}\right) \oplus H_{2(m-n)+1}\left(M, \mathbb{Z}_{2}\right) !
$$

For example, this splitting occurs if the normal bundle of $M$ admits a nonvanishing section. It follows from the Gysin sequence that this condition is satisfied if the codimension of $M \subset W$ is greater than its dimension $(n>m)$, (see, for example, [8, Section 11]).

The following is our main result:

Theorem 1.3 Suppose that $(W, \Omega)$ is geometrically bounded and symplectically aspherical. Let $M \subset W$ be a closed symplectic submanifold whose unit normal bundle is homologically trivial in degree $2 m$. Then, for all sufficiently small $R>0$, the symplectic tubular neighborhood $U_{R}$ has refined capacity

$$
c_{\mathrm{HZ}}^{1,2 \pi R^{2}}\left(U_{R}\right)=\pi R^{2} .
$$

\footnotetext{
${ }^{1}$ For simplicity, group isomorphisms will always be denoted with an "=" sign.
} 
The monotonicity property of the capacity implies the following corollary.

Corollary 1.4 For $(W, \Omega)$ and $M$ as in Theorem 1.3 every open set $U \subset$ $U_{R} \subset W$ has $c_{\mathrm{HZ}}^{1,2 \pi R^{2}}(U) \leq \pi R^{2}$.

Remark 1.5 The size of the values of $R$ for which our method of proof for Theorem 1.3 works is restricted by two factors. First, we are required to consider small enough neighborhoods of $M$ on which $\Omega$ has a standard normal form. More importantly, even for trivial tubular neighborhoods, we need $\pi R^{2}$ to be less than the Gromov width of $(M, \omega)$.

\subsection{Other recent results}

We now describe the content of some other recent works [5, 12, 26, 50, 59] which also consider the capacity of symplectic tubular neighborhoods.

As part of his work in [5], Biran finds an upper bound for the Gromov width of symplectic tubular neighborhoods of certain closed codimension-two symplectic submanifolds of closed Kähler manifolds.

The papers [12, 26, 50, all consider various relative capacities of symplectic tubular neighborhoods. The monotonicity property of these relative capacities applies to small open sets which contain $M$. This is adequate to obtain new almost/dense existence results for certain Hamiltonian flows which describe the classical motion of a charged particle in a nondegenerate magnetic field (see Section 2.2.).

In [12] and 26], the ambient manifold $(W, \Omega)$ is assumed to be geometrically bounded and symplectically aspherical. The paper [12] considers a relative homological symplectic capacity which is defined as in $[6]$. For symplectic tubular neighborhoods $U_{R}$ with sufficiently small radius, it is shown that this relative capacity equals $\pi R^{2}$. In [26], the authors consider a relative version of the Hofer-Zehnder capacity which is defined using test Hamiltonians that attain their maximum value in an open neighborhood of the symplectic submanifold. They prove that the relative Hofer-Zehnder capacity of $U_{R}$ is also equal to $\pi R^{2}$. This allows the authors to improve the dense existence results from 12 . to almost existence results.

In [50, Macarini introduces a stabilization procedure which allows him to bound the capacity of a symplectic manifold $(W, \Omega)$ which admits a free Hamiltonian circle action in terms of the capacity of compact subsets of $W \times T^{*} S^{1}$. These sets 
can be symplectically embedded into a trivial symplectic tubular neighborhood $W \times B^{2}(R)$ whose capacity is known to be finite in various cases by [36, 48, 49, [53. When applied to $U_{R} \backslash M$ this yields bounds on certain relative capacities and allows Macarini to further improve the existence results from [12, 26] by relaxing the assumption that the ambient manifold is symplectically aspherical.

Most recently, in 59, Schlenk has obtained a powerful new generalization of Hofer's energy-capacity inequality from [33, where the standard capacity is replaced by the contractible capacity and the displacement energy is replaced by the stable displacement energy. The proof involves Macarini's stabilization procedure as well as several techniques and theorems from the study of Hofer's geometry on the space of Hamiltonian diffeomorphisms.

Schlenk uses his energy-capacity inequality, together with the results of Laudenbach, Polterovich and Sikorav on the displacement of subsets of symplectic manifolds, to obtain the following result:

Theorem 1.6 (Schlenk [59]) Let $(W, \Omega)$ be a symplectic manifold which is geometrically bounded and stably strongly semi-positive. Let $M$ be any closed submanifold of $W$ such that either $\operatorname{dim} M<\operatorname{codim} M$, or $\operatorname{dim} M=\operatorname{codim} M$ and $M$ is not Lagrangian. Then every sufficiently small tubular neighborhood of $M$ has finite (contractible) Hofer-Zehnder capacity.

The hypotheses of this theorem are surprisingly mild and consequently, when $\operatorname{dim} M \leq \operatorname{codim} M$, it has much broader applications to Hamiltonian dynamics than one obtains from the bounds on capacities from [12, 26, 50] or Theorem 1.3 .

On the other hand, the more restrictive assumptions of Theorem 1.3 allow us

to use the refined capacity $c_{\mathrm{Hz}}^{1, \kappa}$. The almost existence theorem for this capacity includes bounds on the symplectic area of periodic orbits (Theorem 2.2), and in certain cases these bounds can be used to obtain stronger existence theorems, (Lemma 2.3).

\subsection{Comparison of techniques}

Outside of the techniques developed by Hofer and Zehnder for $\left(\mathbb{R}^{2 l}, \Omega_{2 l}\right)$, there are three general methods known to the author for bounding Hofer-Zehnder capacities.

The first of these methods was introduced in [20] and developed in [36] (see also [47, 48, 49, 53]). This method utilizes Floer's version of Gromov's compactness 
theorem for perturbed $J$-holomorphic spheres. Roughly speaking, one starts with a test Hamiltonian $H$ and a nonempty moduli space $\mathcal{S}$ of regular $J_{-}$ holomorphic spheres with marked points which get mapped to $x_{0} \in\{H=0\}$ and $x_{\max } \in\{H=\max (H)\}$. Then, the test Hamiltonian $H$ is used to produce the family of perturbed Cauchy-Riemann equations $\bar{\partial}_{J}+\nabla(\lambda H)=0$, where $\lambda \geq 0$. One can then show that: the collection of moduli spaces $\left(\lambda, \mathcal{S}_{\lambda}\right)$ is noncompact; $\mathcal{S}_{\lambda}$ is nonempty for small $\lambda>0$; and $\mathcal{S}_{\lambda}$ is empty for all $\lambda$ greater than some positive constant (which bounds the capacity from above). Under suitable assumptions the source of noncompactness can then be identified as convergence to a broken Floer cylinder which breaks along a nonconstant orbit of some $\lambda H$.

In the present work, we use a squeezing argument in Floer homology from [26]. This is complementary to the previous method since it works best in the absence of holomorphic spheres and relies on Floer's gluing theorem as well as his compactness result. The general idea is to use the algebraic framework of filtered Floer homology to detect nontrivial orbits. The origins of the strategy can be found in the computations of Symplectic Homology in 19] (see also [6. 12]). In [26, these ideas were distilled by Ginzburg and Gürel into an effective method for finding upper bounds for Hofer-Zehnder capacities.

To illustrate the basic principle, consider a test function $H$ on a set $U$. The function $H$ can be approximated from above and below by two model Hamiltonians $G_{+}$and $G_{-}$, respectively, whose dynamics (and Floer homology) is completely understood. One then considers the monotone Floer continuation maps

$$
\sigma_{G_{-} G_{+}}: \mathrm{HF}^{a, b}\left(G_{+}\right) \rightarrow \mathrm{HF}^{a, b}\left(G_{-}\right),
$$

for action values $a>\max (H)$. Floer's compactness and gluing theorems imply that this map must factor through $\operatorname{HF}^{a, b}(H)$. Hence, if one can show that $\sigma_{G_{-} G_{+}}$is nontrivial, then it follows that $\operatorname{HF}^{a, b}(H) \neq 0$ and $H$ must have a 1 -periodic orbit with action greater than $\max (H)$. As described above, such an orbit must be nonconstant. In this way, one obtains an algebraic version of the existence problem. However, there are simple examples where the map $\sigma_{G_{-} G_{+}}$is trivial for all $a>\max (H)$. Moreover, even when $\sigma_{G_{-} G_{+}}$is nontrivial, the precise transfer mechanism is often quite subtle.

The results from [6, 12, 19, 26, are all established using this type of argument. In these papers, it is possible for the authors to work at the homology level because the model Hamiltonians $G_{+}$and $G_{-}$have qualitatively similar dynamics on the relevant level sets. In the present work, this is not the case (the relevant level sets are not even diffeomorphic). To overcome this difficulty we are required to also work at the chain level in Floer theory. 
For a description of the methods from Hofer's geometry used by Schlenk in [59, the reader is referred to the paper itself as well as to the references [39, 57. We only mention here that at the center of the argument lies the main result from [53] which gives a sufficient condition, in terms of periodic orbits, for a Hamiltonian $H$ to generate a length minimizing path with respect to Hofer's norm. In turn, the main result in 55] is implied by an upper bound for a particular Hofer-Zehnder capacity of trivial symplectic tubular neighborhoods [44. It is a remarkable fact that all the finiteness theorems for capacities implied by Schlenk's Theorem 1.6 can be traced to this one bound for the capacity of trivial symplectic tubular neighborhoods.

\subsection{Extensions of Theorem 1.3}

We now describe how some of the hypotheses of Theorem 1.3 can be relaxed.

Most importantly, it should be possible to relax the hypothesis that $(W, \Omega)$ is symplectically aspherical and instead assume that the minimal Chern number is sufficiently large and there exists a symplectic area gap, ie

$$
\inf _{A \in \pi_{2}(W)}\left\{\left|\int_{A} \Omega\right|: \int_{A} \Omega \neq 0\right\}>0 .
$$

One can then repeat the chain level arguments described here using the FloerNovikov complex from [34, which also comes with a filtration by the action functional. In particular, as described above, the proof of Theorem 1.3 relies on an analysis of the Floer complexes of two model Hamiltonians restricted to a specific action interval, say $(a, b)$. The orbits of these model Hamiltonians come with natural choices of spanning discs. By choosing $b-a$ to be sufficiently small, the area gap (and the appropriate lower bound for the minimal Chern number) allows one to ignore all other choices of spanning discs.

It will also be clear from the proof that Theorem 1.3 holds for the Hofer-Zehnder capacity which is defined using time dependent test Hamiltonians $H:[0,1] \times$ $W \rightarrow \mathbb{R}$ such that $H$ has compact support in $[0,1] \times U_{R}$ and each function $H_{t}=$ $H(t, \cdot)$ attains its maximum value on a common set with nonempty interior. (Such functions play a prominent role in 60]).

Remark 1.7 The possible applications of the squeezing method for computing new bounds for Hofer-Zehnder capacities are restricted primarily by the fact that standard Floer theory counts only perturbed holomorphic cylinders (twice punctured spheres) and hence can only see periodic orbits in one homotopy class. In a joint project with V L Ginzburg and B Gürel [27] we will construct 
a more versatile version of Floer theory following the blueprint of Symplectic Field Theory [14].

\subsection{Organization of the paper}

In the next section, we describe some applications of Theorem 1.3 to classical Hamiltonian flows and Hofer's geometry. The remainder of the paper is devoted to the proof of Theorem 1.3. In Section 3, we define symplectic tubular neighborhoods and reduce Theorem 1.3 to a result about the dynamics of test Hamiltonians on these neighborhoods, Theorem 3.3. The necessary tools from Floer theory are then described in Section 4. In Section 5, we prove Theorem 3.3 by reducing it to a sequence of statements about the nontriviality of monotone Floer continuation maps which are then proved using the tools from Section 4. Appendix $\mathrm{A}$ contains the proof of an upper bound for the area of planar curves with positive curvature. This bound is needed for the applications in Section 2. Finally, a proof of a well-known result for the Morse homology of fibre-bundles is contained in Appendix B.

\subsection{Acknowledgements}

The author would like to express his profound gratitude to Viktor Ginzburg for many valuable conversations concerning this work. He is also grateful to Stephanie Alexander, Dusa McDuff, and Felix Schlenk for their helpful comments and suggestions. The author would also like to thank the referee of this paper for the extremely thorough and insightful report. Most of this work was undertaken and completed during the author's stays at Stony Brook University and Walailak University. He thanks these institutions for their warm hospitality and generous support. This research was partially supported by NSF Grant DMS-0405994.

\section{Applications}

\subsection{An almost existence theorem with area bounds}

When one can prove that the Hofer-Zehnder capacity of a symplectic manifold is finite, there are remarkable consequences for the autonomous Hamiltonian flows on the manifold. In particular, one obtains the almost existence theorem of 
Hofer-Zehnder and Struwe [38, 64. We recall the statement of this result below and show that the corresponding result for the refined capacity $c_{\mathrm{HZ}}^{1, \kappa}$ includes bounds on the symplectic area of periodic orbits. In fact, we will use a slight generalization of the almost existence theorem due to Macarini and Schlenk [51.

Let $S \subset(W, \Omega)$ be a hypersurface, ie an oriented closed submanifold of codimension one. The restriction of $\Omega$ to $S$ has a one-dimensional kernel which determines the characteristic foliation of $S$. A closed characteristic of $S$ is an embedding of the circle into $S$ whose image is a closed leaf of this foliation.

If $S$ is a regular level set of a Hamiltonian $H$, then the periodic orbits of $H$ on $S$ are in one-to-one correspondence with the closed characteristics of $S$. As demonstrated by the counterexamples in 223, 24, 25, 31, 42, not every regular level set (hypersurface) carries a periodic orbit (closed characteristic). However it is still fruitful to study the existence question near a fixed hypersurface $S$. Following [37, one does this by considering a thickening of $S$. This is a diffeomorphism $\psi:(-1,1) \times S \rightarrow W$ onto an open and bounded neighborhood of $S$ such that $\psi(0, \cdot): S \rightarrow W$ is the inclusion map. In other words, $\psi$ determines a family of hypersurfaces $S_{r}=\psi(\{r\} \times S)$ which are modeled on $S$.

Theorem 2.1 (Hofer-Zehnder [38, Macarini-Schlenk [51] and Struwe 64]) Let $U$ be a neighborhood of a hypersurface $S$ in $(W, \Omega)$ such that $c_{\mathrm{HZ}}(U)<\infty$. If $\psi$ is a thickening of $S$ whose image is contained in $U$, then there is a closed characteristic on $S_{r}$ for almost every $r \in(-1,1)$.

The almost existence theorem corresponding to the refined capacity $c_{\mathrm{HZ}}^{1, \kappa}$ includes bounds on the symplectic area.

Theorem 2.2 Let $S$ be a hypersurface of a weakly exact symplectic manifold $(W, \Omega)$, and let $U$ be a neighborhood of $S$ such that $c_{\mathrm{Hz}}^{1, \kappa}(U)<\infty$ for some $\kappa<$ $\infty$. If $\psi$ is a thickening of $S$ with image in $U$, then almost every hypersurface $S_{r}$ carries a contractible closed characteristic $x$ with symplectic area satisfying $|\mathcal{A}(x)| \leq c_{\mathrm{HZ}}^{1, \kappa}(U)+\kappa$.

Proof This result follows easily from the arguments in [38, 64] and their refinements in [51]. The key point is that during the usual limit process, outlined below, one can use the bounds on the action given by the capacity $c_{\mathrm{HZ}}^{1, \kappa}$ to obtain the bounds on the symplectic area.

The limit process from [38, 51, 64] is structured as follows. For a fixed $r \in$ $(-1,1)$ one constructs a sequence of test Hamiltonians $F_{i}$ on $U$ such that 
$\max \left(F_{i}\right)>c_{\mathrm{Hz}}(U)$. It follows that each $F_{i}$ has a nonconstant periodic orbit $y_{i}$ on some level $S_{r_{i}}$. The functions $F_{i}$ are constructed so that the $r_{i}$ converge to $r$. To prove the almost existence theorem, one then shows that the $y_{i}$ converge to a periodic orbit $x$ on $S_{r}$ for almost all values of $r$.

If this procedure is repeated using the refined capacity $c_{\mathrm{HZ}}^{1, \kappa}$, we obtain a sequence of nonconstant contractible periodic orbits $y_{i}$ with period equal to one, such that

$$
\max \left(F_{i}\right)<\int_{0}^{1} F_{i}\left(y_{i}(t)\right) d t-\mathcal{A}\left(y_{i}\right)<\max \left(F_{i}\right)+\kappa .
$$

Since each $F_{i}$ is nonnegative and $\int_{0}^{1} F_{i}\left(y_{i}(t)\right) d t<\max \left(F_{i}\right)$, this implies that

$$
0>\mathcal{A}\left(y_{i}\right)>-\max \left(F_{i}\right)-\kappa .
$$

The $F_{i}$ can also be chosen so that $\max \left(F_{i}\right)-c_{\mathrm{HZ}}^{1, \kappa}>0$ is arbitrarily small. In particular, for a sequence $\epsilon_{i} \rightarrow 0^{+}$we have

$$
0>\mathcal{A}\left(y_{i}\right) \geq-c_{\mathrm{HZ}}^{1, \kappa}-\kappa-\epsilon_{i} .
$$

Now, when the sequence of orbits $y_{i}$ converges, the limit $x \subset S_{r}$ is a nontrivial contractible periodic orbit with period equal to one and symplectic area satisfying

$$
0 \geq \mathcal{A}(x) \geq-c_{\mathrm{HZ}}^{1, \kappa}-\kappa .
$$

The existence question for thickenings of a hypersurface was first considered for $\left(\mathbb{R}^{2 l}, \Omega_{2 l}\right)$ by Hofer and Zehnder in [37. There the authors establish the existence of closed characteristics on a dense set of hypersurfaces in a thickening, and they also obtain the same information about the symplectic area of these characteristics. Since [37] predates Floer's creation of his homology theory, it goes without saying that the methods used in 37] are much different from those used here.

As noted in [37, the bounds on the symplectic area can be used, in the presence of certain a priori bounds, to prove the existence of periodic orbits on fixed hypersurfaces (see also 3, 2]). More precisely, consider a situation where Theorem 2.2 holds. That is, we have a hypersurface $S$ and a constant $K>0$ such that for any suitable thickening $\psi$ almost every hypersurface $S_{r}$ of $\psi$ contains a contractible closed characteristic $x$ with $|\mathcal{A}(x)| \leq K$.

Lemma 2.3 37 Suppose there is a thickening $\psi$ of $S$ and a constant $C>0$ such that for any closed characteristic $y$ in the image of $\psi$ we have

$$
|\mathcal{A}(y)| \geq C l(y)
$$

where $l(y)$ is the length of $y$ with respect to some fixed metric. Then $S$ carries a contractible closed characteristic $x$ with $|\mathcal{A}(x)| \leq c_{\mathrm{HZ}}^{1, \kappa}(U)+\kappa$. 
Proof By Theorem 2.2 we can find a sequence $r_{i} \rightarrow 0$ such that there is a closed characteristic $y_{i}$ on $S_{r_{i}}$ whose areas are uniformly bounded from above. The a priori bounds in Lemma 2.3 yield a uniform upper bound for the lengths of the $y_{i}$. Since the lengths of closed characteristics are also uniformly bounded away from zero [37, the result follows immediately from the Arzela-Ascoli Theorem.

If $S$ is a regular level set of some Hamiltonian $H$ and the thickening $\psi$ is determined by the nearby level sets of $H$, then the bound in Lemma [2.3 can be replaced by one involving the period instead of the length. In particular it suffices to find a constant $C$ such that for any periodic orbit $y$ of $H$ with period $T$ we have

$$
|\mathcal{A}(y)| \geq C T \text {. }
$$

\subsection{Classical Hamiltonian flows}

Using Theorem 2.2 and Lemma2.3, we describe in this section some applications of Theorem 1.3 to classical Hamiltonian flows.

Let $(M, g)$ be a Riemannian manifold equipped with a closed two-form $\sigma$ and a function $V: M \rightarrow \mathbb{R}$ which has a minimum value of zero. To this data we associate the Hamiltonian flow on the cotangent bundle $\pi: T^{*} M \rightarrow M$ defined by the twisted symplectic form

$$
\Omega_{\sigma}=d \lambda+\pi^{*} \sigma
$$

and the total energy Hamiltonian $H_{V}: T^{*} M \rightarrow \mathbb{R}$ given, in canonical $q, p-$ coordinates, by

$$
H_{V}(q, p)=\frac{1}{2} g^{-1}(q)(p, p)+V(q) .
$$

The flow of $H_{V}$ with respect to $\Omega_{\sigma}$ describes the classical motion of a charged particle on $M$ under the influence of the magnetic field $\sigma$ and the (electrostatic) force with potential $V$. For simplicity we will refer to this as the electromagnetic flow for the pair $(\sigma, V)$.

The problem of establishing the existence of periodic orbits for these flows has been studied extensively. When $V=0$ and $\sigma=0$ this corresponds to the famous problem of finding closed geodesics for $(M, g)$. When $\sigma=0$, Bolotin proved the existence of closed orbits on every regular level set in [7]. When $V=0$, it is known from 22 that there may exist regular energy levels without periodic orbits. However, for these purely magnetic flows, it is believed that 
closed orbits exist on all small energy levels. Indeed, this has been established in many cases, (see, for example, 11, 9, 21, 41]).

Schlenk's recent work leads to the following almost existence result in this setting.

Theorem 2.4 (Schlenk [59]) For the electro-magnetic flow determined by a pair $(\sigma, V)$ with $\sigma \neq 0$, there are contractible periodic orbits on almost every sufficiently small energy level.

From Theorem 2.2 and Theorem 1.3 we immediately obtain the following refinement of this result under several extra hypotheses.

Theorem 2.5 Consider the electro-magnetic flow determined by a pair $(\sigma, V)$ where the magnetic two-form is nondegenerate. Suppose that $(M, \sigma)$ is symplectically aspherical and the unit normal bundle of $M$ in $T^{*} M$ is homologically trivial in degree $\operatorname{dim}(M)$. Then there is a number $K>0$ such that almost every sufficiently small energy level carries a contractible periodic orbit with symplectic area less than $K$.

Remark 2.6 The conditions on $(M, \sigma)$ are automatically satisfied in the important case when $(M, \sigma)$ is an even-dimensional torus with any symplectic form.

\subsection{Example: nondegenerate magnetic fields on the flat torus}

According to Lemma 2.3 the area bounds in Theorem 2.5 can sometimes be used to pass from almost existence to genuine existence results. To the knowledge of the author, the only cases where this strategy has been used to obtain existence results is for classical flows with no magnetic term $(\sigma=0)$, see 37, 35, 39]. Below we show that this strategy can also be used to partially recover the existence results of Arnold from [1] for nondegenerate magnetic flows on the flat torus.

Let $\mathbb{T}^{2}$ be the two-dimensional torus equipped with its standard flat metric. For angular coordinates $q_{1}$ and $q_{2}$ on the torus, we let $p_{1}$ and $p_{2}$ denote the conjugate momenta so that $\left(q_{1}, q_{2}, p_{1}, p_{2}\right)$ are global coordinates on $T^{*} \mathbb{T}^{2}$. We then consider magnetic two-forms of the form

$$
\sigma=F\left(q_{1}, q_{2}\right) d q_{1} \wedge d q_{2}
$$


where the function $F$ is nonvanishing and positive. The corresponding Hamiltonian is the kinetic energy

$$
H=\frac{1}{2}\left(p_{1}^{2}+p_{2}^{2}\right) .
$$

If we set $p_{1}=r \cos \theta$ and $p_{2}=r \sin \theta$, then the dynamics on the level $\{H=E\}$ is described by the following deceptively simple system of equations

$$
\dot{q}_{1}=\sqrt{2 E} \cos \theta, \quad \dot{q_{2}}=\sqrt{2 E} \sin \theta, \quad \dot{\theta}=-F\left(q_{1}, q_{2}\right) .
$$

The fact that $F$ is nonvanishing can be used to establish the existence of periodic orbits on fixed energy levels. One way to do this is to note that if $F$ is nonvanishing, then $\theta$ is strictly decreasing under the flow. Following [1, this allows one to define, for every energy value $E$, a Poincaré return map $\psi_{E}: \mathbb{T}^{2} \rightarrow \mathbb{T}^{2}$ whose fixed points correspond to periodic orbits on the level $\{H=E\}$. In particular, if $\phi^{t}$ denotes the flow on $\{H=E\}$, then $\psi_{E}\left(q_{1}, q_{2}\right)$ is defined by the equation

$$
\phi^{t}\left[\left(q_{1}, q_{2}\right), 0\right]=\left[\psi_{E}\left(q_{1}, q_{2}\right),-2 \pi\right] .
$$

In 1, Arnold observes that the return maps $\psi_{E}$ are all Hamiltonian diffeomorphisms of $\mathbb{T}^{2}$, (see [46] for more details). Applying Conley and Zehnder's proof of the Arnold Conjecture for symplectic tori [13], he then obtains:

Theorem 2.7 (Arnold 1) For a nondegenerate magnetic field on the flat torus there are at least three distinct contractible periodic orbits on every level set and at least four if they are nondegenerate.

For nondegenerate magnetic flows in higher dimensions, these return maps do not exist and there is not yet a way to generalize Arnold's argument. We now show that it is possible to partially recover Arnold's result by using the fact that $F$ does not vanish to establish the a priori bounds required in Lemma 2.3 It is hoped that this strategy can also be used in higher dimensions.

To begin, we note that when Theorems 1.3 and 2.2 are applied to the magnetic flows above we get:

Proposition 2.8 For a nondegenerate magnetic two-form $\sigma=F\left(q_{1}, q_{2}\right) d q_{1} \wedge$ $d q_{2}$ on the flat torus there is a $K>0$ such that almost every low energy level carries a contractible periodic orbit $x$ with symplectic area $|\mathcal{A}(x)|<K$.

At this point we observe that since $F$ is nonvanishing, the projection to $\mathbb{T}^{2}$ of a closed trajectory of (2) is a closed curve with strictly negative curvature. This observation can be used to obtain the desired a priori area bounds when the relative variance of $F$ is small. More precisely, let $\bar{F}$ and $\underline{F}$ denote the maximum and minimum values of $F$, respectively, and set $V_{F}=\overline{\bar{F}} / \underline{F}$. 
Proposition 2.9 If $V_{F}<\sqrt{\frac{\pi}{2}}$, then there is a continuous function $C(E)>0$ such that

$$
C(E) T \leq|\mathcal{A}(x)| .
$$

for every periodic orbit $x$ of (2) with period $T$ and energy $E$.

By Lemma 2.3, we will then have

Theorem 2.10 Let $\sigma=F\left(q_{1}, q_{2}\right) d q_{1} \wedge d q_{2}$ be a nondegenerate magnetic twoform on the flat torus such that

$$
V_{F}<\sqrt{\frac{\pi}{2}}
$$

For the corresponding magnetic flow, there is a contractible periodic orbit on every sufficiently low energy level. Moreover, the symplectic areas of these orbits are uniformly bounded.

Remark 2.11 The orbits detected in Theorems 2.7 and 2.10 may be different. In particular, Theorem 2.7 finds closed orbits on $\{H=E\}$ which wrap once around the fibre, whereas the orbits detected by Theorem 2.10] are distinguished only by their symplectic area.

Proof of Proposition 2.9 Let $x(t)$ be a periodic orbit of (2) with period $T$ and energy $E$. Let $\gamma(t)$ denote the projection of $x(t)$ to $\mathbb{T}^{2}$.

The symplectic area $\mathcal{A}(x)$ can be divided into two terms

$$
\begin{aligned}
\mathcal{A}(x) & =\int_{D^{2}} \bar{x}^{*} \Omega_{\sigma} \\
& =\int_{D^{2}} \bar{x}^{*} d \lambda+\int_{D^{2}} \bar{x}^{*}\left(\pi^{*} \sigma\right) \\
& =\int_{D^{2}} \bar{x}^{*} d \lambda+\int_{D^{2}} \bar{\gamma}^{*} \sigma \\
& =\mathcal{A}_{1}(x)+\mathcal{A}_{2}(\gamma)
\end{aligned}
$$

A simple computation yields

$$
\mathcal{A}_{1}(x)=2 E T \text {. }
$$

Now let $k=\frac{1}{2 \pi} \int_{0}^{T} \dot{\theta}(t) d t$ be the total rotation number of $\gamma$. It is clear from (2), that

$$
\underline{F T} \leq-2 \pi k \leq \bar{F} T
$$


Hence,

$$
\mathcal{A}_{1}(x) \geq-k(4 E \pi / \bar{F}) .
$$

To bound $\mathcal{A}_{2}(\gamma)$ from below, we note that (2) implies that the projection $\gamma(t)$ has constant speed $\sqrt{2 E}$ and negative curvature equal to $-F(\gamma(t)) / \sqrt{2 E}$.

Proposition 2.12 Let $\xi:[0, T] \rightarrow \mathbb{R}^{2}$ be a closed planar curve with constant speed $v$, rotation number $k$, and positive curvature $K(t) / v$ such that $0<\underline{K} \leq$ $K(t)$. The Euclidean area enclosed by $\xi, A(\xi)$, satisfies

$$
0 \leq A(\xi) \leq k 4(v / \underline{K})^{2} .
$$

The proof of this result is elementary and is included in Appendix A.

Applying Proposition 2.12 to the curve $\xi(t)=\gamma(T-t)$ we get the following bounds for the Euclidean area enclosed by $\gamma$,

$$
0 \geq A(\gamma) \geq k\left(8 E / \underline{F}^{2}\right) .
$$

For $\mathcal{A}_{2}(\gamma)$, the area of $\gamma$ with respect to $\sigma$, we then get

$$
\mathcal{A}_{2}(\gamma) \geq k \bar{F}\left(8 E / \underline{F}^{2}\right)
$$

Taken together, inequalities (3), (4) and (5) imply that

$$
\begin{aligned}
\mathcal{A}(x) & \geq-k\left(\frac{4 E \pi}{\bar{F}}-\frac{8 E \bar{F}}{\underline{F}^{2}}\right) \\
& =-k \frac{8 E}{\bar{F}}\left(\frac{\pi}{2}-V_{F}^{2}\right) \\
& \geq T \frac{4 E}{\pi V_{F}}\left(\frac{\pi}{2}-V_{F}^{2}\right) \\
& =T C(E) .
\end{aligned}
$$

Clearly, $C(E)$ is positive for $1 \leq V_{F}<\sqrt{\frac{\pi}{2}}$ and Proposition 2.9 follows.

\subsection{Hofer's Geometry and $c_{\mathrm{HZ}}^{1, \infty}$}

Theorem 1.3 can also be used to obtain new lower bounds for other symplectic invariants of symplectic tubular neighborhoods. These invariants are defined in terms of Hofer's geometry on the group of Hamiltonian diffeomorphisms.

Recall that each smooth, time-dependent, compactly supported Hamiltonian $H \in C_{c}^{\infty}([0,1] \times W)$ determines a Hamiltonian flow, $\phi_{H}^{t \in[0,1]}$, on $(W, \Omega)$. The group of Hamiltonian diffeomorphisms of $(W, \Omega), \operatorname{Ham}(W, \Omega)$, consists of all the 
time -1 maps $\phi_{H}^{1}$ obtained in this manner. Conversely, for every path $\phi^{t \in[0,1]}$ in $\operatorname{Ham}(W, \Omega)$ there is a Hamiltonian $H \in C_{c}^{\infty}([0,1] \times W)$ which generates it, ie $\phi^{t}=\phi_{H}^{t} \circ \phi^{0}$. When $\left(W^{2 l}, \Omega\right)$ is closed, this generating Hamiltonian is uniquely determined by the normalization condition

$$
\int_{W} H(t, x) \Omega^{l}=0, \text { for all } t \in[0,1] .
$$

Otherwise, the generating Hamiltonian is already uniquely determined by the fact that it has compact support.

In 32, Hofer defines the length of a Hamiltonian path $\phi^{t}$, in terms of its generating Hamiltonian $H$, by the formula

$$
\begin{aligned}
\operatorname{Length}\left(\phi^{t}\right) & =\int_{0}^{1} \max _{x \in W} H(t, x) d t-\int_{0}^{1} \min _{x \in W} H(t, x) d t \\
& =\operatorname{Length}^{+}\left(\phi^{t}\right)+\operatorname{Length}^{-}\left(\phi^{t}\right) .
\end{aligned}
$$

The Hofer metric on $\operatorname{Ham}(W, \Omega)$ is then defined by

$$
\rho(\phi, \psi)=\inf \operatorname{Length}\left(\phi^{t}\right),
$$

where the infimum is taken over all smooth paths joining $\phi$ to $\psi$. A path $\phi^{t \in[0,1]}$ is said to be length minimizing if $\operatorname{Length}\left(\phi^{t}\right)=\rho\left(\mathrm{id}, \phi^{1}\right)$.

The quantity Length ${ }^{+}\left(\phi^{t}\right)$ is called the positive Hofer length of $\phi^{t}$, and one defines the positive Hofer metric $\rho^{+}$in the obvious way.

One of the central problems in the study of Hofer's geometry is to characterize the Hamiltonian paths which minimize the (positive) Hofer length. This question is profoundly related to the dynamical features of the corresponding flow [56, 57. It is of particular interest for paths which are generated by time-independent Hamiltonians (referred to here as autonomous Hamiltonian paths), because these describe important dynamical systems from classical mechanics. The first result in this direction was proved by Hofer in 33. There he shows that an autonomous Hamiltonian path in $\operatorname{Ham}\left(\mathbb{R}^{2 l}, \Omega_{2 l}\right)$ is length minimizing as long as its flow has no nonconstant periodic orbits with period less than or equal to one. This result has been extended in several directions 44, 43, 44, 53, 52, 54, 62, 65] and Polterovich has conjectured that it is true for general symplectic manifolds [57].

In [63], Sikorav shows that the length minimizing property of an autonomous Hamiltonian path is also constrained by the set on which it is supported. This motivates the definition of the following notion, which we call the Hofer width of an open subset $U$ of $(W, \Omega)$ :

$$
w_{H}(U):=\sup \left\{\max (H)-\min (H) \mid H \in C_{c}^{\infty}(U), \operatorname{Length}\left(\phi_{H}^{t}\right)=\rho\left(\mathrm{id}, \phi_{H}^{1}\right)\right\} .
$$


This invariant measures the size of $U$ in $W$ in terms of the length of the longest autonomous Hamiltonian path which is supported in $U$ and is length minimizing in $\operatorname{Ham}(W, \Omega)$.

The positive Hofer width $w_{H}^{+}(U)$, is defined analogously as follows

$$
w_{H}^{+}(U):=\sup \left\{\max (H) \mid H \in C_{c}^{\infty}(U), H \geq 0, \text { Length }^{+}\left(\phi_{H}^{t}\right)=\rho^{+}\left(\mathrm{id}, \phi_{H}^{1}\right)\right\} .
$$

When $W$ is noncompact, the positive Hofer width $w_{H}^{+}(U)$ can be interpreted in the same way as $w_{H}(U)$. When $W$ is closed this interpretation must be altered slightly, because the quantity $\max (H)$ is no longer equal to the positive length of $\phi_{H}^{t}$. In particular, each $H \in C_{c}^{\infty}(W)$ is not properly normalized when $W$ is closed, and

$$
\operatorname{Length}^{+}\left(\phi_{H}^{t}\right)=\max (H)-\frac{1}{\operatorname{volume}(W)} \int_{W} H(t, x) \Omega^{l} .
$$

Regardless of this, it is evident from (6) that $\max (H)$ is a reasonable substitute for the positive Hofer length since

$$
\max (H) \geq \operatorname{Length}^{+}\left(\phi_{H}^{t}\right) \geq \max (H)(1-\operatorname{volume}(U) / \operatorname{volume}(W)) .
$$

For a closed symplectic manifold $(W, \Omega)$, the work of McDuff [52] implies that the (positive) Hofer width of any open subset $U \subset W$ is nonzero. For tubular neighborhoods of certain submanifolds much more can be said. For example, suppose that in addition to being closed, $(W, \Omega)$ is symplectically aspherical. Let $L \subset W$ be a Lagrangian submanifold such that the map $\pi_{1}(L) \rightarrow \pi_{1}(W)$ (induced by inclusion) is injective, and $L$ admits a metric $g$ with no contractible geodesics, eg $L$ is a torus or admits a hyperbolic metric. In this case, it follows from the work of Schwarz 60 that the (positive) Hofer width of any neighborhood of $L$ is infinite. In particular, it is easy to construct a positive autonomous Hamiltonian $H$ supported in any neighborhood of $L$, such that $\max (H)$ is arbitrarily large and the flow of $H$ has no nonconstant contractible periodic orbits of any period. Schwarz proves in 60] that the latter property implies that $\phi_{H}^{t}$ minimizes the (positive) Hofer length.

In 43], this sufficient condition for a Hamiltonian path to be length minimizing is refined. The following result can be easily derived from [43, Theorem 1.8].

Proposition 2.13 (Kerman-Lalonde [43]) Let $H$ belong to $\mathcal{H}_{\text {test }}(U)$ and suppose that the flow of $H$ has no nonconstant contractible periodic orbits with period equal to one and action greater than $\max (H)$. Then the path $\left\{\phi_{H}^{t}\right\}_{t \in[0,1]}$ minimizes the positive Hofer length. 
In other words, every admissible test Hamiltonian used to define $c_{\mathrm{HZ}}^{1, \infty}(U)$ generates a Hamiltonian path which minimizes the positive Hofer length. From this, we immediately get the following inequality.

Proposition 2.14 Suppose that $(W, \Omega)$ is closed and symplectically aspherical. For every open set $U$ in $W$ we have

$$
w_{H}^{+}(U) \geq c_{\mathrm{HZ}}^{1, \infty}(U) .
$$

Theorem 1.3 now implies the following result.

Theorem 2.15 For any symplectic tubular neighborhood $U_{R}$ as in Theorem 1.3, we have

$$
w_{H}^{+}\left(U_{R}\right) \geq \pi R^{2}
$$

Remark 2.16 Instead of the Hofer width of $U$, one may consider the extrinsic diameter of $\operatorname{Ham}(U, \Omega)$, ie the diameter of $\operatorname{Ham}(U, \Omega)$ as a subset of $\operatorname{Ham}(W, \Omega)$. This is clearly bounded from below by the positive Hofer width. However, Ostrover [55] has recently proven that if $W$ is closed and $\pi_{2}(W)=0$, then the extrinsic diameter of $\operatorname{Ham}(U, \Omega)$ is infinite for any open set $U \subset W$ The intrinsic diameter of $\operatorname{Ham}(U, \Omega)$ is unlikely to be interesting, as Polterovich has conjectured that the diameter of $\operatorname{Ham}(W, \Omega)$ for any symplectic manifold is infinite [56].

Remark 2.17 Using Proposition 2.14, we can generalize the discussion above concerning the Hofer width of neighborhoods of Lagrangian submanifolds. Let $L$ be a Lagrangian submanifold of $(W, \Omega)$ which we again assume is closed and symplectically aspherical. Let $g$ be any metric on $L$. By Weinstein's Neighborhood theorem there is a neighborhood $V_{R}$ of $L$ in $W$ which is symplectomorphic to a neighborhood of the zero-section in $\left(T^{*} L, d \lambda\right)$ of the form $\left\{(q, p) \in T^{*} L \mid\|p\|_{g}^{2}=R^{2}\right\}$. Set $T(g, L, W)$ equal to the minimal period of all closed geodesics of $g$ which represent a homotopy class in the kernel of the map $\pi_{1}(L) \rightarrow \pi_{1}(W)$. A simple argument shows that $c_{\mathrm{HZ}}^{1, \infty}\left(V_{R}\right) \geq T(g, L, W) R$. Hence, by Proposition 2.14 $w_{H}^{+}\left(V_{R}\right) \geq T(g, L, W) R$.

\section{Symplectic tubular neighborhoods}

In this section we define the symplectic tubular neighborhoods $U_{R}$ of $M$ in $(W, \Omega)$. We also show that there are Hamiltonian flows on each $U_{R}$ which

\footnotetext{
${ }^{2}$ The author thanks the referee for informing him of this point.
} 
are totally periodic. These flows are then used to obtain lower bounds for the capacity of $U_{R}$. This allows us to reduce the proof of Theorem 1.3 to a statement concerning the dynamics of test Hamiltonians on symplectic tubular neighborhoods.

\subsection{The definition}

Let $\pi: E \rightarrow M$ be the symplectic normal bundle to $M$, ie the symplectic orthogonal complement to $T M$ in $\left.T W\right|_{M}$. Henceforth, we will identify $M$ with the zero-section of $E$ and write points in $E$ as pairs $(p, z)$, where $p \in M$ and $z$ is in the fibre of $E$ over $p$. The bundle $E$ is a symplectic vector bundle with a fibrewise symplectic form $\sigma_{E}$ given by the restriction of $\Omega$ to the fibres. Let $J_{E}$ be a fibrewise almost complex structure on $E$ which is compatible with $\sigma_{E}$. This yields a fibrewise inner product $g_{E}(\cdot, \cdot)=\sigma_{E}\left(\cdot, J_{E} \cdot\right)$ and we denote the function $(p, z) \mapsto g_{E}(p)(z, z)$ by the (over-simplified) notation $\|z\|^{2}$. For these choices, we set

$$
U_{R}=\left\{(p, z) \in E \mid\|z\|^{2} \leq R^{2}\right\} .
$$

On $E \backslash M$ there is a canonical fibrewise one-form $\alpha_{E}$ which is defined by the formula

$$
\alpha_{E}(p, z)(\cdot)=\|z\|^{-2} \sigma_{E}(p)(z, \cdot)
$$

and satisfies

$$
d^{F}\left(\frac{1}{2}\|z\|^{2} \alpha_{E}\right)=\sigma_{E},
$$

where $d^{F}$ denotes the fibrewise exterior differential. Fixing a Hermitian connection $\nabla$ on $E$ we can extend $\alpha_{E}$ to a genuine one-form on $E \backslash M$ which we denote by $\alpha$. We now consider the closed two-form

$$
d\left(\frac{1}{2}\|z\|^{2} \alpha\right)+\omega
$$

which is defined on all of $E$ and is nondegenerate, and hence symplectic, on a neighborhood of the zero section. Here we have identified $\omega=\left.\Omega\right|_{M}$ with its pull-back to the total space $E$.

By Weinstein's Symplectic Neighborhood Theorem, $\left(U_{R}, d\left(\frac{1}{2}\|z\|^{2} \alpha\right)+\omega\right)$ is symplectomorphic to a neighborhood of $M$ in $(W, \Omega)$ when $R>0$ is sufficiently small. We will restrict our attention to values of $R$ for which this symplectomorphism exists 3 We refer to these neighborhoods as symplectic tubular neighborhoods of $M$.

\footnotetext{
${ }^{3}$ This is the first condition restricting the size of $R$.
} 


\subsection{Dynamics on $U_{R}$}

Lemma 3.1 On each $U_{R}$, the Hamiltonian flow of the function $\|z\|^{2}$ with respect to the symplectic form $\Omega=d\left(\frac{1}{2}\|z\|^{2} \alpha\right)+\omega$ is periodic with period $\pi$.

Proof Let $V_{\text {Hopf }}(p, z)=J_{E}(p) z$ be the vector field on $E$ which generates the standard fibrewise Hopf circle action with period $2 \pi$. Then

$$
\begin{aligned}
i_{V_{\mathrm{Hopf}}}\left(d\left(\frac{1}{2}\|z\|^{2} \alpha\right)+\omega\right) & =i_{V_{\mathrm{Hopf}}}\left(d\left(\frac{1}{2}\|z\|^{2} \alpha\right)\right) \\
& =\mathcal{L}_{V_{\mathrm{Hopf}}}\left(\frac{1}{2}\|z\|^{2} \alpha\right)-d\left(i_{V_{\mathrm{Hopf}}}\left(\frac{1}{2}\|z\|^{2} \alpha\right)\right) \\
& =-d\left(\frac{1}{2}\|z\|^{2}\right),
\end{aligned}
$$

which proves the result.

Corollary 3.2 The capacity $c_{\mathrm{HZ}}\left(U_{R}\right)$ is no less than $\pi R^{2}$.

Proof Let $h_{\epsilon}:\left[0, R^{2}\right] \rightarrow[0, \infty)$ be a smooth function satisfying

- $h_{\epsilon}(s)=\pi R^{2}-\epsilon$ for $s$ near 0 ,

- $h_{\epsilon}(s)=0$ for $s$ near $R^{2}$,

- $\left|h_{\epsilon}^{\prime}\right|<\pi$ for all $s \in\left[0, R^{2}\right]$.

Consider the test Hamiltonian $H_{\epsilon}(p, z)=h_{\epsilon}\left(\|z\|^{2}\right)$ on $U_{R}$. The Hamiltonian vector field of $H_{\epsilon}$ is $X_{H_{\epsilon}}=2 h_{\epsilon}^{\prime}\left(\|z\|^{2}\right) \cdot V_{\text {Hopf }}$. Since $\left|h_{\epsilon}^{\prime}\right|<\pi$, it follows that $H_{\epsilon}$ is admissible for all $\epsilon>0$.

The previous corollary also implies that $c_{\mathrm{HZ}}^{1,2 \pi R^{2}}\left(U_{R}\right) \geq \pi R^{2}$, and so to prove Theorem 1.3 we must show that $c_{\mathrm{HZ}}^{1,2 \pi R^{2}}\left(U_{R}\right) \leq \pi R^{2}$. This upper bound for the refined capacity is implied by the following result concerning the dynamics of test Hamiltonians on $U_{R}$.

Theorem 3.3 Suppose that $(W, \Omega)$ is geometrically bounded and symplectically aspherical. Let $M^{2 m}$ be a closed symplectic submanifold of $W$ whose unit normal bundle is homologically trivial in degree $2 m$. For all sufficiently small $R>0$, if $H \in \mathcal{H}_{\text {test }}\left(U_{R}\right)$ satisfies $\max (H)>\pi R^{2}$, then $H$ has a nonconstant 1 -periodic orbit with action in the interval $\left(\max (H), \max (H)+2 \pi R^{2}\right)$.

The rest of this paper is devoted to the proof of Theorem 3.3 


\section{Tools from Floer theory}

To prove Theorem 3.3, we will use machinery from Floer theory which takes advantage of the filtration by the action functional. In this section we recall the definitions and properties of the necessary tools when $(W, \Omega)$ is geometrically bounded and symplectically aspherical. For such manifolds this material is essentially standard by now and we recall the required results without proof. The reader is referred to the sources [10, 15, 16, 17, 18, 34, 58, 61] for more details.

In fact, we make use of the Morse complex, the Floer complex for Hamiltonian diffeomorphisms, and the Floer complex for pairs of Lagrangians. We begin by describing why all three of these objects are used in the proof.

Recall that a Morse complex can be associated to a generic choice of a Morse function $h$ and a metric $g$ on a closed manifold. The Morse chain complex $\left(C(h), \partial_{g}\right)$ consists of the vector space $C(h)$ generated by the critical points of $h$ and the boundary map $\partial_{g}$ which counts trajectories of the negative gradient flow of $h$ with respect to $g$. The homology of this complex is independent of the choice of the data $h$ and $g$ and is isomorphic to the homology of the manifold on which they are defined.

Let $\mathcal{H}=C_{c}^{\infty}\left(S^{1} \times W\right)$ be the space of smooth, time-periodic, compactly supported functions on a symplectic manifold $(W, \Omega)$. Every $G \in \mathcal{H}$ determines an action functional on the space of smooth contractible loops in $W$. As well, each $S^{1}$-family of $\Omega$-compatible almost complex structures, $J_{t}$, determines a metric on this loop space. The Hamiltonian Floer complex for a generic pair $\left(G, J_{t}\right)$ is then defined, at least heuristically, as the Morse complex for this functional and metric on the loop space.

Alternatively, the same data, $G$ and $J_{t}$, determines a $[0,1 / 2]--$ family of almost complex structures $\widetilde{J}_{t}^{W}$ on the product manifold

$$
(\widetilde{W}, \widetilde{\Omega}):=(W \times W, \Omega \oplus(-\Omega)) .
$$

This family can then be used to define a Lagrangian Floer complex for the pair of Lagrangian submanifolds of $\widetilde{W}$ consisting of the diagonal $\Delta$ and the graph of $\phi_{G}^{1}$.

These two Floer complexes associated to $G$ and $J_{t}$ are identical (see Section 4.5). However, in the Lagrangian setting one has the added freedom to compute the homology by replacing the graph of $\phi_{G}^{1}$ and $\widetilde{J}_{t}^{W}$ by a Lagrangian submanifold and family of almost complex structures which aren't necessarily 
determined by objects on $W$. This freedom will allow us to make some crucial identifications of certain restricted Lagrangian Floer complexes with Morse complexes. This is achieved using the Morse-Bott version of Lagrangian Floer theory developed by Poźniak in [58, (see Theorem 4.1). It is not clear whether these identifications can be obtained using Hamiltonian Floer theory alone (cf [6. 11]).

In the first part of this section we recall the necessary material from Lagrangian Floer theory, including Poźniak's theory of clean intersections from [58. We also discuss the definitions and properties of monotone Floer continuation maps. In the second part of the section we recall the basic construction of the Hamiltonian Floer complex and we describe the natural translation from Hamiltonian Floer theory to Lagrangian Floer theory. Finally, we describe some useful algebraic relations satisfied by monotone Floer continuation maps in the Hamiltonian setting.

Concerning notation In what follows, objects defined on (for) the manifold $\widetilde{W}$ will be decorated with a tilde. If, in addition, they are defined using an object coming from $W$, then they will also be given a superscript $W$. Generic approximations of any object will be denoted by a prime.

\subsection{Lagrangian Floer homology}

We describe here the simplest version of Lagrangian Floer theory which is defined for a fixed Lagrangian submanifold and its images under Hamiltonian diffeomorphisms. We will restrict our attention to the Lagrangian submanifold given by the diagonal $\Delta$ in the product manifold $(\widetilde{W}, \widetilde{\Omega})=(W \times W, \Omega \oplus(-\Omega))$.

It is clear from the definitions that if $(W, \Omega)$ is symplectically aspherical and geometrically bounded with respect to the almost complex structure $J_{g b}$, then $(\widetilde{W}, \widetilde{\Omega})$ is also symplectically aspherical and is geometrically bounded with respect to the almost complex structure $\widetilde{J}_{g b}=J_{g b} \oplus-J_{g b}$. Let

$$
\widetilde{\mathcal{H}}=C_{c}^{\infty}\left(S^{1} \times \widetilde{W}, \mathbb{R}\right)
$$

be the space of smooth time-periodic Hamiltonians on $\widetilde{W}$ which have compact support. Given a $\widetilde{G} \in \widetilde{\mathcal{H}}$, we define $\widetilde{\mathcal{J}}_{[0,1 / 2]}=\widetilde{\mathcal{J}}_{[0,1 / 2]}(\widetilde{G})$ to be the set of all smooth $[0,1 / 2]$-families of $\widetilde{\Omega}$-tame almost complex structures $\widetilde{\mathcal{J}}_{t}$ on $\widetilde{W}$ which are $\widetilde{\Omega}$-compatible near the support of $\widetilde{G}$ and are equal to $\widetilde{J}_{g b}$ outside some compact set. For a generic pair $\left(\widetilde{G}, \widetilde{J}_{t}\right) \in \widetilde{\mathcal{H}} \times \widetilde{\mathcal{J}}_{[0,1 / 2]}$, the corresponding 
Lagrangian Floer chain complex for actions in the positive interval $(a, b)$ is denoted by

$$
\left(\mathrm{CF}^{a, b}(\Delta, \widetilde{G}), \partial_{\widetilde{J_{t}}}\right),
$$

and is constructed as follows.

Let $\widetilde{\mathcal{L}}=\widetilde{\mathcal{L}}(\widetilde{G})$ denote the space of smooth paths

$$
\left\{\widetilde{x} \in C^{\infty}([0,1 / 2], \widetilde{W}) \mid \widetilde{x}(0) \in \Delta, \widetilde{x}(1 / 2) \in\left(\phi_{\widetilde{G}}^{1}\right)^{-1}(\Delta)\right\} .
$$

Consider the action functional $\widetilde{\mathcal{A}}_{\widetilde{G}}: \widetilde{\mathcal{L}} \rightarrow \mathbb{R}$ defined by

$$
\widetilde{\mathcal{A}}_{\widetilde{G}}(\widetilde{x})=-\int_{[0,1 / 2]^{2}} \widetilde{v}^{*} \widetilde{\Omega}
$$

where $\widetilde{v}$ is a map from $[0,1 / 2] \times[0,1 / 2]$ to $\widetilde{W}$ such that $\widetilde{v}(s, \cdot) \in \widetilde{\mathcal{L}}$ for all $s \in[0,1 / 2], \widetilde{v}(1 / 2, t)=\widetilde{x}(t)$, and $\widetilde{v}(0, t)=\gamma_{0}(t)$ for some fixed $\gamma_{0} \in \widetilde{\mathcal{L}}$. The critical point set of $\widetilde{\mathcal{A}}_{\widetilde{G}}, \operatorname{Crit}\left(\widetilde{\mathcal{A}}_{\widetilde{G}}\right)$, consists of the constant paths in $\widetilde{\mathcal{L}}$ and hence coincides with the intersection points of the diagonal $\Delta$ and its image $\left(\phi_{\widetilde{G}}^{1}\right)^{-1}(\Delta)$. A critical point $\widetilde{x}$ is nondegenerate if the corresponding point of intersection is transverse. In this case, $\widetilde{x}$ has a well-defined Maslov index, $\mu_{\text {Maslov }}(\widetilde{x}, \widetilde{G})$.

For constants $0<a<b$, let $\widetilde{\mathcal{H}}^{a, b} \subset \widetilde{\mathcal{H}}$ be the open set of all functions $\widetilde{G}$ in $\widetilde{\mathcal{H}}$ such that $a$ and $b$ are not critical values of $\widetilde{\mathcal{A}}_{\widetilde{G}}$. For $\widetilde{G} \in \widetilde{\mathcal{H}}^{a, b}$, let $\operatorname{Crit}^{a, b}\left(\widetilde{\mathcal{A}}_{\widetilde{G}}\right)$ be the set of critical points of $\widetilde{\mathcal{A}}_{\widetilde{G}}$ with action in the interval $(a, b)$. Generically, the elements of $\operatorname{Crit}^{a, b}\left(\widetilde{\mathcal{A}}_{\widetilde{G}}\right)$ are nondegenerate and hence finite in number. In this case, the Lagrangian Floer chain group of $\widetilde{G}$ for actions restricted to $(a, b)$ is defined to be the graded $\mathbb{Z}_{2}$-vector space

$$
\mathrm{CF}^{a, b}(\Delta, \widetilde{G})=\bigoplus_{\tilde{x} \in \mathrm{Crit}^{a, b}\left(\tilde{\mathcal{A}}_{\tilde{G}}\right)} \mathbb{Z}_{2} \widetilde{x} .
$$

The Floer boundary operator $\partial_{\widetilde{J}_{t}}$ is defined using the Floer moduli spaces $\widetilde{\mathcal{M}}\left(\widetilde{x}, \widetilde{y}, \widetilde{G}, \widetilde{J}_{t}\right)$ for pairs $\widetilde{x}, \widetilde{y} \in \mathrm{Crit}^{a, b}\left(\widetilde{\mathcal{A}}_{\widetilde{G}}\right)$. Each $\widetilde{\mathcal{M}}\left(\widetilde{x}, \widetilde{y}, \widetilde{G}, \widetilde{J_{t}}\right)$ consists of the maps $\widetilde{u}: \mathbb{R} \times[0,1 / 2] \rightarrow \widetilde{W}$ which are solutions of the equation

$$
\partial_{s} \widetilde{u}+\widetilde{J}_{t}(\widetilde{u}) \partial_{t} \widetilde{u}=0
$$

satisfy the boundary conditions

$$
\widetilde{u}(s, 0) \in \Delta \quad \text { and } \quad \widetilde{u}(s, 1 / 2) \in\left(\phi_{\widetilde{G}}^{1}\right)^{-1}(\Delta),
$$

and have limits

$$
\lim _{s \rightarrow-\infty} \widetilde{u}(s, t)=\widetilde{x}(t) \quad \text { and } \quad \lim _{s \rightarrow \infty} \widetilde{u}(s, t)=\widetilde{y}(t),
$$


which are uniform in $t$. For generic data $\widetilde{G}$ and $\widetilde{J}_{t}$, every moduli space $\widetilde{\mathcal{M}}\left(\widetilde{x}, \widetilde{y}, \widetilde{G}, \widetilde{J}_{t}\right)$ is a smooth manifold of dimension $\mu_{\text {Maslov }}(\widetilde{x}, \widetilde{G})-\mu_{\text {Maslov }}(\widetilde{y}, \widetilde{G})$. (If $\widetilde{G}$ is fixed, then any family $\widetilde{J}_{t}$ which forms a generic data pair with $\widetilde{G}$ will be said to be regular for $\widetilde{G}$.) We also note that each $\widetilde{\mathcal{M}}\left(\widetilde{x}, \widetilde{y}, \widetilde{G}, \widetilde{J}_{t}\right)$ is equipped with a free $\mathbb{R}$-action given by $\tau \cdot \widetilde{u}(s, t)=\widetilde{u}(s+\tau, t)$.

Let $\operatorname{Crit}_{k}^{a, b}\left(\widetilde{\mathcal{A}}_{\widetilde{G}}\right)$ denote the subset of $\mathrm{Crit}^{a, b}\left(\widetilde{\mathcal{A}}_{\widetilde{G}}\right)$ which consists of critical points with Maslov index equal to $k$. The boundary operator

$$
\partial_{\widetilde{J}_{t}}: \mathrm{CF}^{a, b}(\Delta, \widetilde{G}) \rightarrow \mathrm{CF}^{a, b}(\Delta, \widetilde{G})
$$

is then defined on each $\widetilde{x} \in \mathrm{Crit}^{a, b}\left(\widetilde{\mathcal{A}}_{\widetilde{G}}\right)$ by the formula

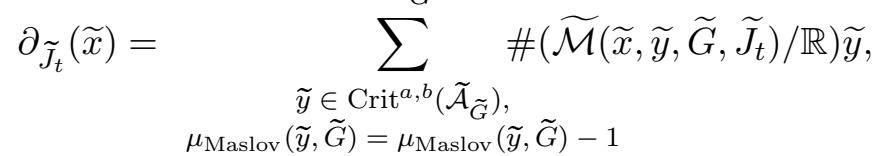

where $\#\left(\widetilde{\mathcal{M}}\left(\widetilde{x}, \widetilde{y}, \widetilde{G}, \widetilde{J}_{t}\right) / \mathbb{R}\right)$ is the number of elements in the 0 -dimensional manifold $\mathcal{M}(\widetilde{x}, \widetilde{y}, \widetilde{G}, \widetilde{J}) / \mathbb{R}$, modulo 2 . Since we are assuming that $(W, \Omega)$ is symplectically aspherical, no bubbling can occur and there are no obstructions. So, the usual arguments imply that

$$
\partial_{\widetilde{J}_{t}} \circ \partial_{\widetilde{J_{t}}}=0 .
$$

The corresponding homology groups $\operatorname{HF}^{a, b}(\Delta, \widetilde{G})$ are independent of the choice of the regular family $\widetilde{J}_{t}$. They are also locally constant on $\widetilde{\mathcal{H}}^{a, b}$. This allows one to define $\operatorname{HF}^{a, b}(\Delta, \widetilde{G})$ for any $\widetilde{G} \in \widetilde{\mathcal{H}}^{a, b}$, regardless of whether or not the elements of $\operatorname{Crit}^{a, b}\left(\widetilde{\mathcal{A}}_{\widetilde{G}}\right)$ are nondegenerate. One just sets $\operatorname{HF}^{a, b}(\Delta, \widetilde{G})=$ $\operatorname{HF}^{a, b}\left(\Delta, \widetilde{G}^{\prime}\right)$ for some $\widetilde{G}^{\prime} \in \widetilde{\mathcal{H}}^{a, b}$ which is close to $\widetilde{G}$ and has the required nondegeneracy property.

\subsection{Clean intersections}

The extension of the definition of the restricted Floer homology $\operatorname{HF}^{a, b}(\Delta, \widetilde{G})$ to every $\widetilde{G} \in \widetilde{\mathcal{H}}^{a, b}$ is particularly useful in the case when $\Delta$ and $\left(\phi_{\widetilde{G}}^{1}\right)^{-1}(\Delta)$ intersect nicely along submanifolds. This situation was first studied by Poźniak in [58] (for more general versions of Lagrangian Floer homology). In this section, we briefly describe Poźniak's results and some refinements from [6], as they apply to our present setting.

A submanifold $\widetilde{N} \subset \Delta \cap\left(\phi_{\widetilde{G}}^{1}\right)^{-1}(\Delta)$ is said to be a clean intersection for $\widetilde{G} \in \widetilde{\mathcal{H}}$ if it is a connected component of $\Delta \cap\left(\phi_{\widetilde{G}}^{1}\right)^{-1}(\Delta)$ and

$$
T_{p}(\widetilde{N})=T_{p}(\Delta) \cap T_{p}\left(\left(\phi_{\widetilde{G}}^{1}\right)^{-1}(\Delta)\right) \text { for all } p \in \widetilde{N} .
$$

For clean intersections, Poźniak proved the following result. 
Theorem 4.1 58, Theorem 3.4.11], 6, Theorem 5.2.2] Suppose that the set $\widetilde{N}$ is a clean intersection for $\widetilde{G} \in \widetilde{\mathcal{H}}^{a, b}$ and that $\widetilde{N}=\operatorname{Crit}^{a, b}\left(\widetilde{\mathcal{A}}_{\widetilde{G}}\right)$. Let $h: \widetilde{N} \rightarrow$ $\mathbb{R}$ be a Morse function and let $g$ be a metric on $\widetilde{N}$ such that the corresponding Morse complex $\left(C(h), \partial_{g}\right)$ is well-defined. Then there is a Hamiltonian $\widetilde{H}_{h} \in \widetilde{\mathcal{H}}$, a family $\widetilde{J}_{g, t} \in \widetilde{\mathcal{J}}_{[0,1 / 2]}$, and a constant $\delta_{0}>0$ such that for every $\delta<\delta_{0}$ we have the following strong equivalence of complexes

$$
\left(\mathrm{CF}^{a, b}\left(\Delta, \widetilde{G}+\delta \widetilde{H}_{h}\right), \partial_{\widetilde{J}_{g, t}}\right) \equiv\left(C(h), \partial_{g}\right) .
$$

The equivalence relation "三" denotes the fact that the elements of the set $\operatorname{Crit}^{a, b}\left(\widetilde{\mathcal{A}}_{\widetilde{G}+\delta \tilde{H}_{h}}\right)$ actually coincide with the critical points of $h$ and the elements of the one-dimensional moduli spaces $\widetilde{\mathcal{M}}\left(\widetilde{x}, \widetilde{y}, \widetilde{G}+\delta \widetilde{H}_{h}, \widetilde{J}_{g, t}\right)$ are $t$-independent and coincide with the negative gradient trajectories of $h$ with respect to $g$.

It follows from (10) that

$$
\operatorname{HF}_{*}^{a, b}(\Delta, \widetilde{G})=H_{*-\mu_{\text {Maslov }}(\widetilde{N}, \widetilde{G})}\left(\widetilde{N} ; \mathbb{Z}_{2}\right) .
$$

We will refer to the grading shift $\mu_{\text {Maslov }}(\widetilde{N}, \widetilde{G})$ as the relative Maslov index of $\widetilde{N}$. It depends on the linearized flow of $\widetilde{G}$ along $\widetilde{N}$.

\subsubsection{Outline of the proof of Theorem 4.1}

To establish the strong equivalence of complexes in equation (10), Poźniak introduces the notion of a local Floer complex of $\widetilde{N}$ and proves that there is a local complex which is strongly equivalent to both sides.

To describe this argument, we first require the notion of a $\widetilde{J}_{t}$-isolating neighborhood of $\widetilde{N}$ in $\widetilde{W}$. This is a precompact neighborhood $\widetilde{U}$ of $\widetilde{N}$ such that any solution $\widetilde{u}: \mathbb{R} \times[0,1 / 2] \rightarrow \widetilde{W}$ of (7) and (8) whose image lies in the closure of $\widetilde{U}$, must satisfy $\widetilde{u}(s, t)=\widetilde{x} \in \widetilde{N}$ for all $(s, t) \in \mathbb{R} \times[0,1 / 2]$. Every clean intersection admits a $\widetilde{J}_{t}$-isolating neighborhood for any choice of the family $\widetilde{J}_{t}$ (see [6, Lemma 5.2.3]).

Let $\widetilde{U}$ be a $\widetilde{J}_{t}$-isolating neighborhood for $\widetilde{N}$ and choose a $\widetilde{G}^{\prime} \in \widetilde{\mathcal{H}}$ such that $\widetilde{G}^{\prime}$ is $C^{2}$-close to $\widetilde{G}$ and the elements of $\operatorname{Crit}\left(\widetilde{\mathcal{A}}_{\widetilde{G}^{\prime}}\right)$ are nondegenerate. The local Floer chain complex of $\widetilde{G}^{\prime}$ is defined to be the $\mathbb{Z}_{2}$-vector space

$$
\mathrm{CF}^{\mathrm{loc}}\left(\widetilde{G}^{\prime}, \widetilde{U}\right)=\bigoplus_{\widetilde{x}^{\prime} \in \operatorname{Crit}\left(\widetilde{\mathcal{A}}_{\widetilde{G}^{\prime}}\right), \widetilde{x}^{\prime} \subset \widetilde{U}} \mathbb{Z}_{2} \widetilde{x}^{\prime} .
$$

For a family $\widetilde{J}_{t}^{\prime}$ which is regular for $\widetilde{G}^{\prime}$ and is $C^{1}$-close to $\widetilde{J}_{t}$, the boundary operator $\partial_{\widetilde{J}_{t}^{\prime}}^{\text {loc }}$ for the complex is defined in the usual way except that it only 
counts solutions $\widetilde{u}$ of (7) whose images lies in $\widetilde{U}$. The corresponding homology is called the local Floer homology of $\widetilde{N}$,

$$
\operatorname{HF}^{\operatorname{loc}}(\widetilde{N}):=H_{*}\left(\mathrm{CF}^{\mathrm{loc}}\left(\widetilde{G}^{\prime}, \widetilde{U}\right), \partial_{\widetilde{J}_{t}^{\prime}}^{\mathrm{loc}}\right),
$$

and Poźniak proves that it is independent of the choices of $\widetilde{U}, \widetilde{G}^{\prime}$ and $\widetilde{J}_{t}^{\prime}$.

To calculate $\operatorname{HF}^{\text {loc }}(\widetilde{N})$, Poźniak starts with a Morse function $h: \widetilde{N} \rightarrow \mathbb{R}$ and a generic metric $g$ on $\widetilde{N}$ and he constructs explicit approximations of $\widetilde{G}$ and $\widetilde{J}_{t}$ whose local Floer complex is strongly equivalent to the Morse complex determined by $h$ and $g$. More precisely, Poźniak proves that there is a Hamiltonian $\widetilde{H}_{h} \in \widetilde{\mathcal{H}}$, a family of almost complex structures $\widetilde{J}_{g, t}$, and a $\delta_{0}>0$ such that for $\delta<\delta_{0}$ one has

$$
\left(\mathrm{CF}^{\mathrm{loc}}\left(\widetilde{G}+\delta \widetilde{H}_{h}, \widetilde{U}\right), \partial_{\widetilde{J}_{g, t}}^{\mathrm{loc}}\right) \equiv\left(C(h), \partial_{g}\right)
$$

for any choice of $\widetilde{U}$ (see [58, Proposition 3.4.6]).

To complete the proof of Theorem 4.1, it only remains to show that if $\widetilde{N}=$ $\operatorname{Crit}^{a, b}\left(\widetilde{\mathcal{A}}_{\widetilde{G}}\right)$ then

$$
\left(\mathrm{CF}^{\mathrm{loc}}\left(\widetilde{G}+\delta \widetilde{H}_{h}, \widetilde{U}\right), \partial_{\widetilde{J}_{g, t}}^{l o c}\right) \equiv\left(\mathrm{CF}^{a, b}\left(\Delta, \widetilde{G}+\delta \widetilde{H}_{h}\right), \partial_{\widetilde{J}_{g, t}}\right)
$$

for sufficiently small $\delta>0$. This follows immediately from [6, Lemma 5.2.4].

\subsection{Monotone Floer continuation maps}

As mentioned above, the restricted Floer homology $\operatorname{HF}^{a, b}(\Delta, \widetilde{G})$ is only locally constant on $\widetilde{\mathcal{H}}^{a, b}$. However, if the functions $\widetilde{G}, \widetilde{H} \in \widetilde{\mathcal{H}}^{a, b}$ satisfy $\widetilde{G} \geq \widetilde{H}$, ie $\widetilde{G}(t, x) \geq \widetilde{H}(t, x)$ for all $(t, x) \in S^{1} \times \widetilde{W}$, then there is a natural homomorphism

$$
\sigma_{\widetilde{H} \widetilde{G}}: \operatorname{HF}^{a, b}(\Delta, \widetilde{G}) \rightarrow \operatorname{HF}^{a, b}(\Delta, \widetilde{H})
$$

called a monotone Floer continuation map. Since our later arguments rely heavily on these maps, we recall their definition.

For $\widetilde{G}, \widetilde{H} \in \widetilde{\mathcal{H}}^{a, b}$ with $\widetilde{G} \geq \widetilde{H}$, a monotone homotopy from $\widetilde{G}$ to $\widetilde{H}$ is a family of functions $\widetilde{G}_{s} \in \widetilde{\mathcal{H}}$ such that $\partial_{s} \widetilde{G}_{s} \leq 0$ and

$$
\widetilde{G}_{s}= \begin{cases}\widetilde{G} & \text { for } s \in(-\infty,-1] \\ \widetilde{H} & \text { for } s \in[1, \infty) .\end{cases}
$$

Given a monotone homotopy $\widetilde{G}_{s}$, let $\widetilde{J}_{s, t}$ be a family of almost complex structures in $\widetilde{\mathcal{J}}_{[0,1 / 2]}$ which is independent of $s$ for all $|s|>1$ such that $\widetilde{J}_{-1, t}$ is regular for $\widetilde{G}$ and $\widetilde{J}_{1, t}$ is regular for $\widetilde{H}$. For each $\widetilde{x} \in \operatorname{Crit}\left(\widetilde{\mathcal{A}}_{\widetilde{G}}\right)$ and $\widetilde{y} \in \operatorname{Crit}\left(\widetilde{\mathcal{A}}_{\widetilde{H}}\right)$, 
let $\widetilde{\mathcal{M}}_{s}\left(\widetilde{x}, \widetilde{y}, \widetilde{G}_{s}, \widetilde{J}_{s, t}\right)$ be the moduli space of maps $\widetilde{u}: \mathbb{R} \times[0,1 / 2] \rightarrow \widetilde{W}$ which are solutions of the equation

$$
\partial_{s} \widetilde{u}+\widetilde{J}_{s, t}(\widetilde{u}) \partial_{t} \widetilde{u}=0,
$$

satisfy the boundary conditions

$$
\widetilde{u}(s, 0) \in \Delta \text { and } \widetilde{u}(s, 1 / 2) \in\left(\phi_{\widetilde{G}_{s}}^{1}\right)^{-1}(\Delta),
$$

and have uniform limits as in (9). For a regular family $\widetilde{J}_{s, t}$, each moduli space $\widetilde{\mathcal{M}}_{s}\left(\widetilde{x}, \widetilde{y}, \widetilde{G}_{s}, \widetilde{J}_{s, t}\right)$ is a smooth manifold of dimension $\mu_{\text {Maslov }}(\widetilde{x}, \widetilde{G})-$ $\mu_{\text {Maslov }}(\widetilde{y}, \widetilde{H})$. The map $\sigma_{\widetilde{H} \widetilde{G}}$ is then defined on each $\widetilde{x} \in \mathrm{Crit}^{a, b}\left(\widetilde{\mathcal{A}}_{\widetilde{G}}\right)$ by

$$
\sigma_{\widetilde{H} \widetilde{G}}(\widetilde{x})=\sum_{\substack{\tilde{y} \in \operatorname{Crit}^{a, b}\left(\widetilde{\mathcal{A}}_{\tilde{H}}\right), \mu_{\text {Maslov }}(\widetilde{y}, \widetilde{H})=\mu_{\text {Maslov }}(\widetilde{x}, \widetilde{G})}} \# \widetilde{\mathcal{M}}_{s}\left(\widetilde{x}, \widetilde{y}, \widetilde{G_{s}}, \widetilde{J}_{s, t}\right) \widetilde{y},
$$

where $\# \widetilde{\mathcal{M}}_{s}\left(\widetilde{x}, \widetilde{y}, \widetilde{G}_{s} \widetilde{J}_{s, t}\right)$ is the number of elements in the zero-dimensional moduli space $\widetilde{\mathcal{M}}_{s}\left(\widetilde{x}, \widetilde{y}, \widetilde{G}_{s}, \widetilde{J}_{s, t}\right)$, modulo 2 .

To prove that $\sigma_{\widetilde{H} \widetilde{G}}$ is a chain map between the restricted Floer complexes above, one must consider the energy of the elements $\widetilde{u} \in \widetilde{\mathcal{M}}_{s}\left(\widetilde{x}, \widetilde{y}, \widetilde{G}_{s}, \widetilde{J}_{s, t}\right)$ which is defined by the formula

$$
E(\widetilde{u})=\int_{0}^{1 / 2} \int_{-\infty}^{+\infty} \widetilde{\Omega}\left(\partial_{s} \widetilde{u}, \widetilde{J}_{s, t}(\widetilde{u}) \partial_{s} \widetilde{u}\right) d s d t
$$

The following useful inequality is a straight forward consequence of Stokes' theorem and the monotonicity assumption on the homotopy $\widetilde{G}_{s}$.

Lemma 4.2 For each $\widetilde{u} \in \mathcal{M}_{s}\left(\widetilde{x}, \widetilde{y}, \widetilde{G}_{s}, \widetilde{J}_{s, t}\right)$ we have:

$$
E(\widetilde{u}) \leq \widetilde{\mathcal{A}}_{\widetilde{G}}(\widetilde{x})-\widetilde{\mathcal{A}}_{\widetilde{H}}(\widetilde{y}) .
$$

Since $E(\widetilde{u}) \geq 0$, inequality (12) implies that the action must decrease under $\sigma_{\tilde{H} \tilde{G}}$. This fact allows one to verify that $\sigma_{\tilde{H} \widetilde{G}}$ is a chain map between the restricted Floer complexes. The homomorphism induced at the level of homology is also denoted by $\sigma_{\widetilde{H} \widetilde{G}}$. It is independent of the choices of the monotone homotopy $\widetilde{G}_{s}$ as well as the family of almost complex structures $\widetilde{J}_{s, t}$.

Remark 4.3 In the description of the map $\sigma_{\widetilde{H} \widetilde{G}}$ we have tacitly assumed the nondegeneracy of the elements of $\operatorname{Crit}^{a, b}\left(\widetilde{\mathcal{A}}_{\widetilde{G}}\right)$ and $\mathrm{Crit}^{a, b}\left(\widetilde{\mathcal{A}}_{\widetilde{H}}\right)$. If some of these intersection points are degenerate, one simply approximates $\widetilde{H}$ and $\widetilde{G}$ by functions $\widetilde{H}^{\prime}$ and $\widetilde{G}^{\prime}$ which have the nondegeneracy property and then 
defines the map between their Lagrangian Floer complexes, as above. By the discussion at the end of Section 4.1 the map induced at the homology level is independent of the approximations $\widetilde{H}^{\prime}$ and $\widetilde{G}^{\prime}$.

The following important property of monotone Floer continuation maps is proved using (12) and the usual compactness and gluing theorems from Floer theory.

Lemma 4.4 Monotone Floer continuation maps satisfy the identities

$$
\begin{aligned}
\sigma_{\widetilde{H} \widetilde{G}} \circ \sigma_{\widetilde{G} \widetilde{F}}=\sigma_{\widetilde{H} \widetilde{F}} \text { for } \widetilde{F} \geq \widetilde{G} \geq \widetilde{H} \\
\sigma_{\widetilde{G} \widetilde{G}}=\text { id for every } \widetilde{G} \in \widetilde{\mathcal{H}}^{a, b} .
\end{aligned}
$$

\subsection{Hamiltonian Floer homology}

Let $\mathcal{H}=C_{c}^{\infty}\left(S^{1} \times W\right)$ be the space of smooth time-periodic Hamiltonians on $W$ which have compact support. For $G \in \mathcal{H}$, let $\mathcal{J}_{S^{1}}=\mathcal{J}_{S^{1}}(G)$ be the set of $S^{1}$-families of $\Omega$-tame almost complex structures on $W$ which are $\Omega$ compatible near the support of $G$ and are equal to $J_{g b}$ outside some compact set. In complete analogy with Section 4.1, one can associate to a generic pair $\left(G, J_{t}\right) \in \mathcal{H} \times \mathcal{J}_{S^{1}}$ and constants $0<a<b$, a restricted Hamiltonian Floer chain complex,

$$
\left(\mathrm{CF}^{a, b}(G), \partial_{J_{t}}\right)
$$

We very briefly describe this complex here and discuss how it can be identified with a Lagrangian Floer chain complex.

Each Hamiltonian $G \in \mathcal{H}$ determines an action functional $\mathcal{A}_{G}: \mathcal{L} \rightarrow \mathbb{R}$ on $\mathcal{L}$, the set of smooth contractible loops in $W$. This is given by the formula

$$
\mathcal{A}_{G}(x)=\int_{0}^{1} G(x(t), t) d t-\int_{D^{2}} v^{*} \Omega
$$

where $v \in C^{\infty}\left(D^{2}, W\right)$ satisfies $\left.v\right|_{\partial D^{2}}=x$. The set of critical points $\operatorname{Crit}\left(\mathcal{A}_{G}\right)$ is precisely the set of contractible 1 -periodic orbits of $G$, and each nondegenerate critical point $x \in \operatorname{Crit}\left(\mathcal{A}_{G}\right)$ has a well-defined Conley-Zehnder index, $\mu_{\mathrm{CZ}}(x, G)$. To accommodate our choice of action functional, we normalize the Conley-Zehnder index here so that a local maximum of a $C^{2}$-small autonomous Hamiltonian on $W^{2 l}$ has Conley-Zehnder index equal to its Morse index minus $l$.

Let $\mathcal{H}^{a, b} \subset \mathcal{H}$ be the set of all $G \in \mathcal{H}$ for which $a$ and $b$ are not critical values of $\mathcal{A}_{G}$. Let $\mathrm{Crit}^{a, b}\left(\mathcal{A}_{G}\right)$ denote the set of critical points of $\mathcal{A}_{G}$ with action 
in the interval $(a, b)$. For a generic $G \in \mathcal{H}^{a, b}$ the elements of $\mathrm{Crit}^{a, b}\left(\mathcal{A}_{G}\right)$ are nondegenerate and the restricted Floer chain group for $G$ is defined to be the finite dimensional, graded $\mathbb{Z}_{2}$-vector space

$$
\mathrm{CF}^{a, b}(G)=\bigoplus_{x \in \mathrm{Crit}^{a, b}\left(\mathcal{A}_{G}\right)} \mathbb{Z}_{2} x .
$$

For a family $J_{t} \in \mathcal{J}_{S^{1}}$ and a pair of critical points $x, y \in \mathrm{Crit}^{a, b}\left(\mathcal{A}_{G}\right)$, the Floer moduli space $\mathcal{M}\left(x, y, G, J_{t}\right)$ consist of the maps $u: \mathbb{R} \times S^{1} \rightarrow M$ that satisfy the equation

$$
\partial_{s} u=J_{t}(u)\left(X_{G}(u)-\partial_{t} u\right),
$$

and have the following limits which are uniform in $t$,

$$
\lim _{s \rightarrow-\infty} u(s, t)=x(t) \quad \text { and } \quad \lim _{s \rightarrow \infty} u(s, t)=y(t) .
$$

If $J_{t}$ is regular, then these moduli spaces are smooth manifolds of dimension $\mu_{\mathrm{CZ}}(x, G)-\mu_{\mathrm{CZ}}(y, G)$ and the Floer boundary operator $\partial_{J_{t}}: \mathrm{CF}^{a, b}(G) \rightarrow$ $\mathrm{CF}^{a, b}(G)$ is defined on the generators $x \in \mathrm{Crit}^{a, b}\left(\mathcal{A}_{G}\right)$ by

$$
\partial_{J_{t}}(x)=\sum_{\substack{y \in \mathrm{Crit}^{a, b}\left(\mathcal{A}_{G}\right), \mu_{\mathrm{CZ}}(y, G)=\mu_{\mathrm{CZ}}(x, G)-1}} \#\left(\mathcal{M}\left(x, y, G, J_{t}\right) / \mathbb{R}\right) y .
$$

Here, $\#\left(\mathcal{M}\left(x, y, G, J_{t}\right) / \mathbb{R}\right)$ is the number, modulo 2 , of elements in the zerodimensional space $\mathcal{M}\left(x, y, G, J_{t}\right) / \mathbb{R}$ where $\mathbb{R}$ acts freely by translation in the $s$-variable.

\subsection{Translating from Hamiltonian to Lagrangian Floer theory}

Given a Hamiltonian $G$ on $W$ we define a corresponding Hamiltonian $\widetilde{G}^{W}$ on $\widetilde{W}$ by the formula

$$
\widetilde{G}^{W}\left(t, x_{0}, x_{1}\right)=G\left(t, x_{0}\right) .
$$

One can easily check that $\left(\phi_{\widetilde{G}^{W}}^{1}\right)^{-1}(\Delta)$ is equal to the graph of $\phi_{G}^{1}$ in $\widetilde{W}$. Note also that

$$
G \geq H \Leftrightarrow \widetilde{G}^{W} \geq \widetilde{H}^{W} .
$$

Now consider the map $\Psi_{G}: \mathcal{L} \rightarrow \widetilde{\mathcal{L}}=\widetilde{\mathcal{L}}\left(\widetilde{G}^{W}\right)$ defined by

$$
x(t) \mapsto \widetilde{x}(t):=\left(\left(\phi_{G}^{t}\right)^{-1}(x(t)), \phi_{G}^{1} \circ\left(\phi_{G}^{1-t}\right)^{-1}(x(1-t))\right),
$$

where the domain of the image is restricted to $[0,1 / 2]$. The composition of $\Psi_{G}$ with $\widetilde{\mathcal{A}}_{\widetilde{G}}$ is equal to $\mathcal{A}_{G}$, ie $\mathcal{A}_{G}(x)=\widetilde{\mathcal{A}}_{\widetilde{G}^{W}}(\widetilde{x})$. In addition, $\Psi_{G}$ identifies the sets $\operatorname{Crit}\left(\mathcal{A}_{G}\right)$ and $\operatorname{Crit}\left(\widetilde{\mathcal{A}}_{\widetilde{G}^{W}}\right)$, and preserves indices. More precisely, $x$ is in 
$\operatorname{Crit}\left(\mathcal{A}_{G}\right)$ if and only if $\widetilde{x}$ is in $\operatorname{Crit}\left(\widetilde{\mathcal{A}}_{\widetilde{G}^{W}}\right)$, and if $x$ is nondegenerate then $\widetilde{x}$ is also nondegenerate with $\mu_{\text {Maslov }}\left(\widetilde{x}, \widetilde{G}^{W}\right)=\mu_{\mathrm{CZ}}(x, G)$.

For a family $J_{t} \in \mathcal{J}_{S^{1}}(G)$, consider the family of $\widetilde{\Omega}$-compatible almost complex structures $\widetilde{J}_{t \in[0,1 / 2]}^{W}$ defined by

$$
\widetilde{J}_{t}^{W}=\left(\left(\phi_{G}^{t}\right)^{*} J_{t}\right) \oplus\left(-\left(\phi_{G}^{1-t} \circ\left(\phi_{G}^{1}\right)^{-1}\right)^{*} J_{1-t}\right) .
$$

The map

$$
u(s, t) \mapsto \widetilde{u}(s, t):=\left(\left(\phi_{G}^{t}\right)^{-1}(u(s, t)), \phi_{G}^{1} \circ\left(\phi_{G}^{1-t}\right)^{-1}(u(s, 1-t))\right),
$$

induced by $\Psi_{G}$, is then a bijection between the moduli spaces $\mathcal{M}\left(x, y, G, J_{t}\right)$ and $\widetilde{\mathcal{M}}\left(\widetilde{x}, \widetilde{y}, \widetilde{G}^{W}, \widetilde{J}_{t}^{W}\right)$, for every pair $x, y \in \operatorname{Crit}\left(\mathcal{A}_{G}\right)$. Thus, for a generic pair $\left(G, J_{t}\right)$, the map $\Psi_{G}$ yields an identification between the Hamiltonian Floer complex $\left(\mathrm{CF}^{a, b}(G), \partial_{J_{t}}\right)$ and the corresponding Lagrangian Floer complex $\left(\mathrm{CF}^{a, b}\left(\Delta, \widetilde{G}^{W}\right), \partial_{\widetilde{J}_{t}^{W}}\right)$. This identification preserves both the action values and indices and yields the following isomorphism in homology

$$
\mathrm{HF}_{*}^{a, b}(G)=\mathrm{HF}_{*}^{a, b}\left(\Delta, \widetilde{G}^{W}\right) .
$$

\subsection{Morse-Bott submanifolds of periodic orbits}

As in the Lagrangian setting, the homology groups $\operatorname{HF}^{a, b}(G)$ corresponding to the complex $\left(\mathrm{CF}^{a, b}\left(G, J_{t}\right), \partial_{J_{t}}\right)$ are independent of the choice of the regular family $J_{t}$ and are locally constant on $\mathcal{H}^{a, b}$. Again, this allows us to define $\operatorname{HF}^{a, b}(G)$ for any $G \in \mathcal{H}^{a, b}$, by approximating $G$ by a Hamiltonian whose relevant 1 -periodic orbits are nondegenerate.

A subset $N \subset \operatorname{Crit}\left(\mathcal{A}_{G}\right)$ is said to be a Morse-Bott manifold of periodic orbits if the set $C_{0}=\{x(0) \mid x \in N\}$ is a closed submanifold of $W$ and $T_{x_{0}} C_{0}=$ $\operatorname{ker}\left(D \phi_{G}^{1}\left(x_{0}\right)-I d\right)$ for every $x_{0} \in C_{0}$.

It follows from [6] that a Morse-Bott manifold $N \subset \mathcal{L}$ of periodic orbits is mapped by $\Psi_{G}$ to a clean intersection $\widetilde{N}$ of $\widetilde{G}^{W}$. The critical submanifold also has a relative Conley-Zehnder index $\mu_{\mathrm{CZ}}(N, G)$ which, by (13) and (11), is equal to $\mu_{\text {Maslov }}\left(\widetilde{N}, \widetilde{G}^{W}\right)$.

\subsection{Monotone Floer continuation maps for Hamiltonian Floer homology}

For $G, H \in \mathcal{H}^{a, b}$ with $G \geq H$ one can define a monotone chain map

$$
\sigma_{H G}: \mathrm{CF}^{a, b}(G) \rightarrow \mathrm{CF}^{a, b}(H),
$$


in the same manner as described in Section 4.3. The translation map $\Psi_{G}$ from Section 4.5 can again be used to identify the maps $\sigma_{H G}$ and $\sigma_{\widetilde{H}^{W} \widetilde{G}^{W}}$ at the chain level. In particular, we have

$$
\sigma_{\widetilde{H}^{W} \widetilde{G}^{W}} \circ \Psi_{G}=\Psi_{G} \circ \sigma_{H G} .
$$

Therefore, the maps $\sigma_{H G}$ satisfy the following version of Lemma 4.4

Lemma 4.5 Monotone Floer continuation maps in Hamiltonian Floer theory satisfy the identities

$$
\begin{aligned}
\sigma_{H G} \circ \sigma_{G F} & =\sigma_{\mathrm{HF}} \text { for } F \geq G \geq H, \\
\sigma_{G G} & =\mathrm{id} \text { for every } G \in \mathcal{H}^{a, b} .
\end{aligned}
$$

The following additional results concerning monotone Floer continuation maps for Hamiltonian Floer homology are well known; see, for example, 10, 18 and [6. Sections 4.4-4.5].

Lemma 4.6 For constants $0<a<b<c$ and a function $G \in \mathcal{H}^{a, b} \cap \mathcal{H}^{b, c}$ the short exact sequence of complexes

$$
0 \rightarrow \mathrm{CF}^{a, b}(G) \rightarrow \mathrm{CF}^{a, c}(G) \rightarrow \mathrm{CF}^{b, c}(G) \rightarrow 0,
$$

yields the exact homology triangle $\triangle_{a, b, c}(G)$ given by

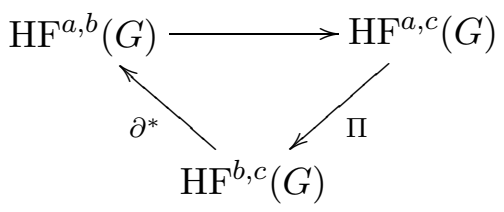

Lemma 4.7 For any $0<a<b<c$ we have the following commuting diagram

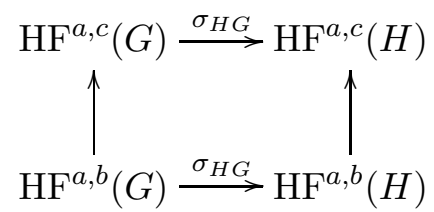

where the vertical arrows are determined by the homology triangles $\triangle_{a, b, c}(G)$ and $\triangle_{a, b, c}(H)$.

Lemma 4.8 If $G_{s}$ is a monotone homotopy from $G$ to $H$ which satisfies $G_{s} \in \mathcal{H}^{a, b}$ for all $s \in[-1,1]$, then $\sigma_{H G}$ is an isomorphism. 
This last result states that the only way in which the map $\sigma_{H G}$ can fail to be an isomorphism is if there is some $s \in(-1,1)$ such that $G_{s}$ has a 1 -periodic orbit with action equal to $a$ or $b$. Even if periodic orbits with action equal to $a$ or $b$ appear during a monotone homotopy, it may still be possible to show that $\sigma_{H G}$ is nontrivial by considering the indices of these orbits. We now describe a useful refinement of Lemma 4.8 which lies at the heart of the calculations in [12, 19, 26].

Definition 4.9 A monotone homotopy $G_{s}$ from $G$ to $H$ is said to be transversal to $(a, b)$ if the following conditions hold.

(1) $G_{s}$ has 1-periodic orbits with action equal to $a$ or $b$ for only a finite set of values $\left\{s_{j}\right\} \subset(-1,1)$.

(2) At each $s_{j}$, these orbits form a Morse-Bott nondegenerate submanifold of 1-periodic orbits, $N_{j}$.

(3) There is an interval $I_{j}=\left(s_{j}-\epsilon_{j}, s_{j}+\epsilon_{j}\right)$ such that $N_{j}$ belongs to a smooth family of Morse-Bott nondegenerate submanifolds $N_{j}(s)$ of $G_{s}$ for $s \in I_{j}$.

(4) The function $\mathcal{A}_{G_{s}}\left(N_{j}(s)\right)$ is continuous and strictly monotone on $I_{j}$.

The space of such homotopies (with $\operatorname{dim}\left(N_{j}\right)=0$ ) is dense in the space of all smooth monotone homotopies from $G$ to $H$.

Proposition 4.10 Let $G_{s}$ be a monotone homotopy from $G$ to $H$ which is transversal to $(a, b)$. Suppose that the relative Conley-Zehnder index of each Morse-Bott nondegenerate submanifold $N_{j}$ is either strictly less than $n_{0}-\operatorname{dim}\left(N_{j}\right)-1$ or strictly greater than $n_{0}+1$. Then the map

$$
\sigma_{H G}: \operatorname{HF}_{n_{0}}^{a, b}(G) \rightarrow \operatorname{HF}_{n_{0}}^{a, b}(H)
$$

is an isomorphism.

Proof In view of Lemma 4.5 we can clearly assume that there is only one $s_{j}$. Consider then, a monotone homotopy $G_{s}$ from $G$ to $H$ which is transverse to $(a, b)$ such that only $G_{s_{1}}$ has 1 -periodic orbits with action equal to $a$ or $b$, and these orbits form a Morse-Bott nondegenerate submanifold $N_{1}$. We assume that $\mathcal{A}_{G_{s_{1}}}\left(N_{1}\right)=b$ and the function $\mathcal{A}_{G_{s}}\left(N_{1}(s)\right)$ is decreasing near $s_{1}$. The other cases can be dealt with in a similar manner.

The set of critical values of $\mathcal{A}_{G_{s_{1}}}, \mathcal{S}\left(G_{s_{1}}\right) \subset \mathbb{R}$, is closed and nowhere dense 60]. It follows from the transversality assumption on $G_{s}$ that there is a $\delta>0$ 
such that the only critical points of $\mathcal{A}_{G_{s_{1}}}$ with action in the interval $[b-\delta, b]$ are those belonging to $N_{1}$.

We also note that the subsets $\mathcal{S}\left(G_{s}\right)$ are lower semi-continuous in $s$ in the following sense: For every open neighborhood $V \subset \mathbb{R}$ of $\mathcal{S}\left(G_{s^{\prime}}\right)$ there is an interval $\left(s^{\prime}-\epsilon, s^{\prime}+\epsilon\right)$ such that $\mathcal{S}_{G_{s}} \subset V$ for all $s \in\left(s^{\prime}-\epsilon, s^{\prime}+\epsilon\right)$ (see [6. Section 4.4]).

It follows that we can choose an $\epsilon>0$ such that for each $s \in\left[s_{1}-\epsilon, s_{1}+\epsilon\right]$ the only critical points of $\mathcal{A}_{G_{s}}$ with action in the interval $[b-\delta, b]$ are those belonging to $N_{1}(s)$. By the transversality assumption, we may also choose $\epsilon$ so that $\mathcal{A}_{G_{s}}\left(N_{1}(s)\right)>b-\delta$ for all $s \in\left[s_{1}-\epsilon, s_{1}+\epsilon\right]$.

We now factor the map $\sigma_{H G}$ as

$$
\sigma_{H G}=\left(\sigma_{H, G_{s_{1}-\epsilon}}\right) \circ\left(\sigma_{G_{s_{1}-\epsilon} G_{s_{1}+\epsilon}}\right) \circ\left(\sigma_{G_{s_{1}+\epsilon} G}\right) .
$$

The first and last terms are isomorphisms by Lemma 4.8. It remains to prove that the middle map is an isomorphism. To do this we consider the commutative diagram from Lemma 4.7

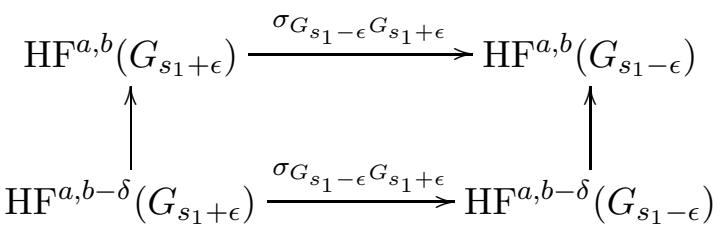

The transversality assumption together with our choices of $\delta$ and $\epsilon$ imply that $G_{s} \in \mathcal{H}^{a, b-\delta}$ for all $s \in\left[s_{1}-\epsilon, s_{1}+\epsilon\right]$. Hence, the bottom map is an isomorphism by Lemma 4.8

The vertical maps belong to the exact sequences determined by Lemma 4.6 for $a<b-\delta<b$. The surrounding terms in these sequences are $\operatorname{HF}_{n_{0}}^{b-\delta, b}\left(G_{s_{1} \pm \epsilon}\right)$ and $\operatorname{HF}_{n_{0}+1}^{b-\delta, b}\left(G_{s_{1} \pm \epsilon}\right)$. Applying Theorem 4.1, we have

$$
\operatorname{HF}_{n_{0}}^{b-\delta, b}\left(G_{s_{1} \pm \epsilon}\right)=H_{n_{0}-\mu_{\mathrm{CZ}}\left(N_{1}, G_{s_{1}}\right)}\left(N_{1}\right)
$$

and

$$
\operatorname{HF}_{n_{0}+1}^{b-\delta, b}\left(G_{s_{1} \pm \epsilon}\right)=H_{n_{0}+1-\mu_{\mathrm{CZ}}\left(N_{1}, G_{s_{1}}\right)}\left(N_{1}\right) .
$$

The assumption that the relative index of $N_{1}$ is greater than $n_{0}+1$ implies that these groups vanish and so the vertical arrows in (15) are also isomorphisms. 


\section{The proof of Theorem 3.3}

For a test Hamiltonian $H \in \mathcal{H}_{\text {test }}\left(U_{R}\right)$, Theorem 3.3 asserts that if $R>0$ is sufficiently small and $\max (H)>\pi R^{2}$, then $H$ has a 1 -periodic orbit with action in the interval $\left(\max (H), \max (H)+2 \pi R^{2}\right)$. To prove this, we will squeeze $H$ between two simple Hamiltonians and study the monotone Floer continuation map between their restricted (Lagrangian) Floer homology groups.

Proposition 5.1 If $R>0$ is sufficiently small, then there are Hamiltonians $G_{+}, G_{-} \in \mathcal{H}$ satisfying $G_{+} \geq H \geq G_{-}$, and constants $a, b$ satisfying

$$
\max (H)<a<b<\max (H)+2 \pi R^{2},
$$

such that the map

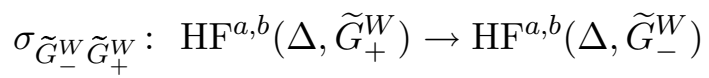

is nontrivial.

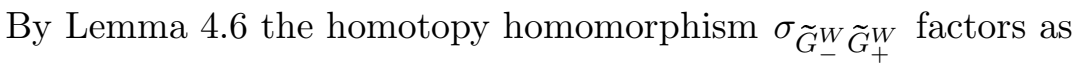

$$
\sigma_{\tilde{G}_{-}^{W} \widetilde{G}_{+}^{W}}=\sigma_{\widetilde{G}_{-}^{W} \widetilde{H}^{W}} \circ \sigma_{\widetilde{H}^{W}} \widetilde{G}_{+}^{W} \cdot
$$

Hence, Proposition 5.1 implies that $\operatorname{HF}^{a, b}\left(\Delta, \widetilde{H}^{W}\right)$ is nontrivial and consequently $\mathrm{CF}^{a, b}\left(\Delta, \widetilde{H}^{W}\right)$ is nonempty. By the translation described in Section 4.5. this, in turn, implies that $\mathrm{CF}^{a, b}(H)$ is nonempty for some $a>\max (H)$. The orbit(s) generating $\mathrm{CF}^{a, b}(H)$ must be nonconstant and so Theorem 3.3 would follow.

To prove Proposition 5.1, we construct a third model Hamiltonian $G_{0} \in \mathcal{H}$ such that $G_{+} \geq G_{0} \geq G_{-}$, and we consider the decomposition

$$
\sigma \widetilde{G}_{-}^{W} \tilde{G}_{+}^{W}=\sigma \widetilde{G}_{-}^{W} \widetilde{G}_{0}^{W} \circ \sigma \widetilde{G}_{0}^{W} \widetilde{G}_{+}^{W} .
$$

We then prove that there are constants $a$ and $b$, satisfying

$$
\max (H)<a<b<\max (H)+2 \pi R^{2},
$$

and a fixed degree $n_{0}$, such that in this degree $\sigma_{\widetilde{G}_{0}^{W}} \widetilde{G}_{+}^{W}$ is a nontrivial surjection and $\sigma_{\widetilde{G}_{-}^{W} \widetilde{G}_{0}^{W}}$ is an isomorphism (see Propositions 5.2 and 5.3 ).

\subsection{The model Hamiltonians}

We now construct the model Hamiltonians $G_{+}, G_{0}$, and $G_{-}$, and describe their Hamiltonian flows. 


\subsubsection{The function $G_{+}$}

The function $G_{+}$is constructed to approximate $H$, from above, near its maximum set. Referring back to the notation of Section 3. $G_{+}$is defined as a reparametrization of the function $\|z\|^{2}$ on $U_{R}$. In particular, we set

$$
G_{+}(p, z)=\alpha\left(\|z\|^{2}\right)
$$

for a smooth nonincreasing function $\alpha$. Since $H$ is in $\mathcal{H}_{\text {test }}\left(U_{R}\right)$, there is an $R^{\prime}<R$ such that $H$ vanishes for $\|z\|>R^{\prime}$. We choose $\alpha$ so that $G_{+}$is constant and equal to its maximum until $\|z\|>R^{\prime}$. We then force $G_{+}$to decrease rapidly to zero at $\|z\|=R$ by choosing the slope of $\alpha$ to decrease rapidly, become constant and finally increase to zero at $R^{2}$. The value of $\max \left(G_{+}\right)$is chosen to be arbitrarily close to, but greater than, $\max (H)$. See Figure 1

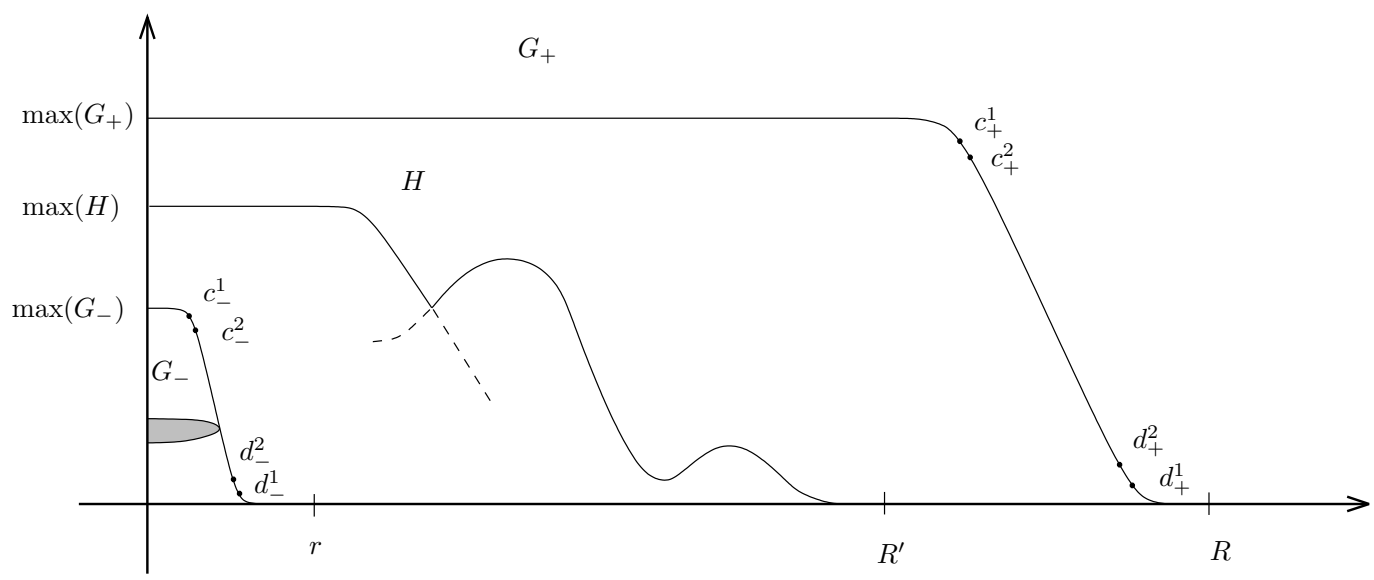

Figure 1: The functions $G_{+}$and $G_{-}$

Since $G_{+}$is a function of $\|z\|^{2}$, its Hamiltonian flow is just a rescaling of the $\pi-$ periodic flow of $\|z\|^{2}$. More precisely, the orbits of $G_{+}$on the level $\|z\|^{2}=c$ are all periodic with period $\pi / \alpha^{\prime}(c)$. When the slope of $\alpha$ is constant and negative we choose it not to be an integer multiple of $\pi$. Hence, the only nonconstant 1-periodic orbits of $G_{+}$are located on two finite sequences of level sets which are labeled in Figure 1. One of these sequences of level sets is located in the region where the slope decreases and the corresponding $G_{+}-$values are denoted by

$$
c_{+}^{1}>c_{+}^{2}>\cdots
$$


The other sequence of 1 -periodic level sets is located in the region where the slope increases. It corresponds to the $G_{+}$-values

$$
d_{+}^{1}>d_{+}^{2}>\cdots
$$

\subsubsection{The function $G_{0}$}

To define $G_{0}$, we first describe the set on which it is supported. Let $p_{\text {fix }}$ be a point on $M \subset W$. We may assume, without loss of generality, that the interior of the set $\{H=\max (H)\}$ intersects the zero section of $U_{R}$ at $\left(p_{\mathrm{fix}}, 0\right)$. This follows from the general fact that given two points of a symplectic manifold and a path between them, there is a Hamiltonian diffeomorphism whose support is close to the path and whose time one flow takes one point to the other along the path. Let $\left(B^{2 m}\left(p_{\text {fix }}, \rho\right), \Omega_{2 m}\right)$ be a Darboux ball in $M$ with radius $\rho$ and center at the point $p_{\text {fix }}$. Here, $\Omega_{2 m}$ denotes the standard symplectic form on $\mathbb{R}^{2 m}$. The bundle $\pi: E \rightarrow M$ is trivial over $B^{2 m}\left(p_{\text {fix }}, \rho\right)$ and we may assume that the connection $\nabla$ is flat over $B^{2 m}\left(p_{\text {fix }}, \rho\right)$. Hence, there is a trivialization of $E$ over $B^{2 m}\left(p_{\text {fix }}, \rho\right)$ for which the symplectic structure $\Omega$ on $\pi^{-1}\left(B^{2 m}\left(p_{\text {fix }}, \rho\right)\right) \cap U_{R}$ has the form

$$
\Omega=\Omega_{2 m} \oplus \Omega_{2 n} .
$$

Assume that $R$ is less than $\rho$ We then define $G_{0}$ by the formula

$$
G_{0}(p, z)=\alpha\left(\|p\|_{2 m}^{2}+\|z\|^{2}\right)
$$

where \|\|$_{2 m}$ is the standard norm on $B^{2 m}\left(p_{\mathrm{fix}}, \rho\right)$, and $\alpha$ is the same function used to define $G_{+}$. Clearly, $G_{+} \geq G_{0}$ and the only place where the functions are equal and both nonzero is on the fibre of $U_{R}$ over $p_{\text {fix }}$. Moreover, $G_{0}$ is supported in the Darboux ball $\mathcal{B}(R):=B^{2(m+n)}\left(\left(p_{\text {fix }}, 0\right), R\right) \subset U_{R}$. See Figure 2,

With the local normal form (16) for $\Omega$, it is also clear that the Hamiltonian flow of the function $\|p\|_{2 m}^{2}+\|z\|^{2}$ with respect to $\Omega$ is $\pi$-periodic on $\mathcal{B}(R)$. Hence, the flow of $G_{0}$ is again just a rescaling of a $\pi$-periodic flow. As above, the nonconstant 1 -periodic orbits of $G_{0}$ are located on two finite sequences of level sets. The $G_{0}$-values of these sequences are denoted by

$$
c_{0}^{1}>c_{0}^{2}>\cdots \quad \text { and } \quad d_{0}^{1}>d_{0}^{2}>\cdots
$$

\footnotetext{
${ }^{4}$ This is the second condition restricting the size of $R$.
} 


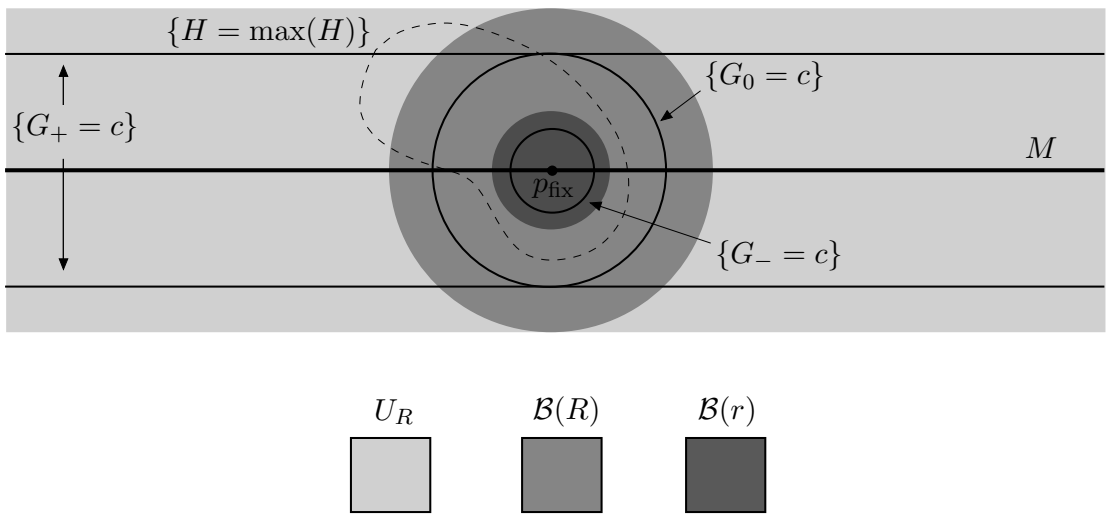

Figure 2: The supports of $G_{+}, G_{0}$ and $G_{-}$

\subsubsection{The function $G_{-}$}

The function $G_{-}$is constructed to approximate $H$, from below, near the point $\left(p_{\text {fix }}, 0\right)$ which we chose to belong to the interior of the set $\{H=\max (H)\}$. Let $\mathcal{B}(r)$ be a Darboux ball with radius $r<R$ and center $\left(p_{\text {fix }}, 0\right)$, where $r$ is small enough so that $\mathcal{B}(r) \subset\{H=\max (H)\}$. We define $G_{-}$by the formula

$$
G_{-}(p, z)=\alpha_{-}\left(\|p\|_{2 m}^{2}+\|z\|^{2}\right) \text {. }
$$

The function $\alpha_{-}$has the same general behavior as $\alpha$. It is chosen so that $G_{-}$ is equal to its maximum in a small neighborhood of $\left(p_{\mathrm{fix}}, 0\right)$ and then decreases to zero within $\mathcal{B}(r)$. When the slope of $\alpha_{-}$is constant and negative we choose it not to be an integer multiple of $\pi$. We also choose $\alpha_{-}$so that $\max \left(G_{-}\right)$ is arbitrarily close to, but smaller than, $\max (H)$. For these choices we have $H \geq G_{-}$(see Figure 1).

Again, the nonconstant 1-periodic orbits of $G_{-}$occur on the level sets corresponding to two finite sequences of $G_{-}-$values, as pictured in Figure 1. We label the two sequences by

$$
c_{-}^{1}>c_{-}^{2}>\cdots \quad \text { and } \quad d_{-}^{1}>d_{-}^{2}>\cdots
$$

\subsubsection{Morse-Bott nondegeneracy}

The functions $\alpha$ and $\alpha_{-}$used to define $G_{+}, G_{0}$, and $G_{-}$are chosen so that their second derivatives are not zero when their slope is nonconstant. This implies that all the nonconstant 1-periodic level sets are Morse-Bott nondegenerate as defined in Section 4.6 (see [6, Lemma 5.3.2]). 


\subsubsection{Actions and indices}

Table 1 summarizes the necessary information concerning the periodic orbits of our model Hamiltonians. For convenience, we will identify a level set $\{G=c\}$ comprised of 1-periodic orbits with its corresponding value $c$. The 1 -periodic orbits on such a level, all have the same action. This common action value is given in the third column of Table 1 up to a term which is denoted by ellipses "..." and can be made arbitrarily small.

\begin{tabular}{|c|c|c|}
\hline Level set, clean int. & Relative index & Action \\
\hline$c_{+}^{k}, \widetilde{c}_{+}^{k}$ & $(2 k-1) n-m+1$ & $\max \left(G_{+}\right)+k \pi R^{2}+\cdots$ \\
$d_{+}^{k}, \widetilde{d}_{+}^{k}$ & $(2 k-1) n-m$ & $k \pi R^{2}+\cdots$ \\
\hline$c_{0}^{k}, \widetilde{c}_{0}^{k}$ & $(2 k-1)(m+n)+1$ & $\max \left(G_{0}\right)+k \pi R^{2}+\cdots$ \\
$d_{0}^{k}, \widetilde{d}_{0}^{k}$ & $(2 k-1)(m+n)$ & $k \pi R^{2}+\cdots$ \\
\hline$c_{-}^{k}, \widetilde{c}_{-}^{k}$ & $(2 k-1)(m+n)+1$ & $\max \left(G_{-}\right)+k \pi r^{2}+\cdots$ \\
$d_{-}^{k}, \widetilde{d}_{-}^{k}$ & $(2 k-1)(m+n)$ & $k \pi r^{2}+\cdots$ \\
\hline
\end{tabular}

Table 1: Actions and indices

Each of the level sets of 1-periodic orbits is nondegenerate in the Morse-Bott sense and so has a relative Conley-Zehnder index. These indices are listed in the second column of Table 1 . For a discussion of the calculation of the relative indices of the level sets of type $c_{+}^{k}$ and $d_{+}^{k}$, the reader is referred to [12, 26]. By construction, the levels of type $c_{0}^{k}, d_{0}^{k}, c_{-}^{k}$ and $d_{-}^{k}$ can be treated as if they are contained in $\mathbb{R}^{2(m+n)}$ and the relative indices can be easily derived from the calculations in [19].

To prove Proposition 5.1 we also need to utilize the corresponding objects in the Lagrangian setting which are obtained using the translation described in Section 4.5. For the translations of the model Hamiltonians we still have

$$
\widetilde{G}_{+}^{W} \geq \widetilde{G}_{0}^{W} \geq \widetilde{G}_{-}^{W}
$$

Since the 1-periodic level sets of the model Hamiltonians are all Morse-Bott nondegenerate, they each get mapped to a clean intersection in $\widetilde{W}$. We denote the clean intersection corresponding to the level $c$ by $\widetilde{c}$, and note that $c$ and $\widetilde{c}$ are diffeomorphic and have the same action value and relative index. For example, the map $\Psi_{G_{+}}$takes $c_{+}^{1}$ to a clean intersection $\widetilde{c}_{+}^{1}$ of $\widetilde{G}_{+}^{W}$, such that every 1 -periodic orbit $x(t) \subset c_{+}^{1}$ gets mapped to the intersection point $(x(0), x(0)) \in \Delta \cap\left(\phi_{\widetilde{G}_{+}^{W}}^{1}\right)^{-1}(\Delta)$. 


\subsection{The constants $a$ and $b$}

We choose the constants $a<b$ so that

$$
\begin{aligned}
\max (H) & <a<\max \left(G_{-}\right)+\pi r^{2}<\max \left(G_{+}\right)+\pi R^{2} \\
& <b<\max \left(G_{+}\right)+2 \pi R^{2} .
\end{aligned}
$$

Since $\max (H)>\pi R^{2}$, the following periodic level sets do not have actions in the interval $(a, b)$ and may be ignored: $d_{+}^{1}, d_{0}^{1}, d_{-}^{1}$, and the levels $c_{+}^{k}$ and $c_{0}^{k}$ for $k \geq 2$.

\subsection{The homomorphisms $\sigma_{\widetilde{G}_{-}^{W} \widetilde{G}_{0}^{W}}$ and $\sigma_{\widetilde{G}_{0}^{W} \widetilde{G}_{+}^{W}}$}

Proposition 5.2 For $a<b$ as in (17) and $n_{0}=m+n+1$, we have

$$
\operatorname{HF}_{n_{0}}^{a, b}\left(\Delta, \widetilde{G}_{0}^{W}\right)=\operatorname{HF}_{n_{0}}^{a, b}\left(\Delta, \widetilde{G}_{-}^{W}\right)=\mathbb{Z}_{2},
$$

and the homomorphism

$$
\sigma_{\widetilde{G}_{-}^{W} \widetilde{G}_{0}^{W}}: \operatorname{HF}_{n_{0}}^{a, b}\left(\Delta, \widetilde{G}_{0}^{W}\right) \rightarrow \operatorname{HF}_{n_{0}}^{a, b}\left(\Delta, \widetilde{G}_{-}^{W}\right)
$$

is an isomorphism.

Proposition 5.3 For $a<b$ as in (17) and $n_{0}=m+n+1$, the homomorphism

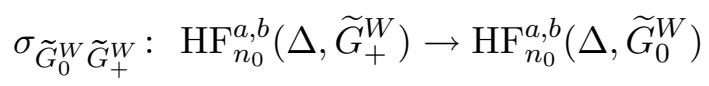

is surjective.

Together, Propositions 5.2 and 5.3 imply that the map

$$
\sigma_{\widetilde{G}_{-}^{W} \widetilde{G}_{+}^{W}}=\sigma_{\widetilde{G}_{-}^{W} \widetilde{G}_{0}^{W}} \circ \sigma_{\widetilde{G}_{0}^{W} \widetilde{G}_{+}^{W}}: \operatorname{HF}_{n_{0}}^{a, b}\left(\Delta, \widetilde{G}_{+}^{W}\right) \rightarrow \operatorname{HF}_{n_{0}}^{a, b}\left(\Delta, \widetilde{G}_{-}^{W}\right)
$$

is nontrivial and Proposition 5.1 follows.

\subsection{Proof of Proposition 5.2}

By the identifications (13) and (14) from Section 4.5] it suffices for us to prove Proposition 5.2 for the corresponding Hamiltonian Floer homology groups. That is, we may prove that

$$
\mathrm{HF}_{n_{0}}^{a, b}\left(G_{0}\right)=\operatorname{HF}_{n_{0}}^{a, b}\left(G_{-}\right)=\mathbb{Z}_{2}
$$

and the map

$$
\sigma_{G_{-} G_{0}}: \operatorname{HF}_{n_{0}}^{a, b}\left(G_{0}\right) \rightarrow \operatorname{HF}_{n_{0}}^{a, b}\left(G_{-}\right)
$$


is an isomorphism.

First we consider the group $\operatorname{HF}_{n_{0}}^{a, b}\left(G_{0}\right)=\operatorname{HF}_{n_{0}}^{a, b}\left(\Delta, \widetilde{G}_{0}\right)$. By our choice of the constants $a<b$ from (17), we know that $\mathrm{Crit}^{a, b}\left(\widetilde{\mathcal{A}}_{\widetilde{G}_{0}^{W}}\right)$ consists of the points in $\widetilde{c}_{0}^{1}$ and possibly one other clean intersection of type $\widetilde{d}_{0}^{k}$ for some $k \geq 2$. We will show that the group $H F_{n_{0}}^{a, b}\left(\Delta, \widetilde{G}_{0}\right)$ is determined solely by $\widetilde{c}_{0}^{1}$. In particular, we show that $H F_{n_{0}}^{a, b}\left(\Delta, \widetilde{G}_{0}\right)$ corresponds, via Theorem 4.1] to $H_{0}\left(\widetilde{c}_{0}^{1} ; \mathbb{Z}_{2}\right)$. The group will be shown not to depend on the intersections of type $\widetilde{d}_{0}^{k}$ with $k \geq 2$, because their relative Maslov indices are too large.

We first note that we may choose the function $G_{0}$ such that the action of $\widetilde{c}_{0}^{1}$ is distinct from the actions of the $\widetilde{d}_{0}^{k}$, for $k \geq 2$. We may then choose constants $a^{\prime}$ and $b^{\prime}$ with $a<a^{\prime}<\widetilde{\mathcal{A}}_{\widetilde{G}_{0}}\left(\widetilde{c}_{0}^{1}\right)<b^{\prime}<b$, such that

$$
\operatorname{Crit}^{a^{\prime}, b^{\prime}}\left(\widetilde{\mathcal{A}}_{\widetilde{G}_{0}^{W}}\right)=\widetilde{c}_{0}^{1} .
$$

Applying Theorem 4.1 to the clean intersection $\widetilde{c}_{0}^{1}$, we get

$$
\operatorname{HF}_{n_{0}}^{a^{\prime}, b^{\prime}}\left(\Delta, \widetilde{G}_{0}\right)=H_{0}\left(\widetilde{c}_{0}^{1} ; \mathbb{Z}_{2}\right)=H_{0}\left(S^{2(m+n)-1} ; \mathbb{Z}_{2}\right)=\mathbb{Z}_{2}
$$

To prove that $\operatorname{HF}_{n_{0}}^{a, b}\left(G_{0}\right)=\mathbb{Z}_{2}$, it remains for us to show that

$$
\operatorname{HF}_{n_{0}}^{a^{\prime}, b^{\prime}}\left(\Delta, \widetilde{G}_{0}\right)=\operatorname{HF}_{n_{0}}^{a, b}\left(\Delta, \widetilde{G}_{0}\right) .
$$

Using the exact triangle from Lemma 4.6 (twice), we see that it suffices to show that the groups $\operatorname{HF}_{*}^{a, a^{\prime}}\left(\Delta, \widetilde{G}_{0}\right)$ and $\operatorname{HF}_{*+1}^{b, b^{\prime}}\left(\Delta, \widetilde{G}_{0}\right)$ are trivial for $*=n_{0}$ and $*=n_{0}-1$.

As described above, for the action window determined by $a$ and $b$, the only way in which the groups $\operatorname{HF}_{*}^{a, a^{\prime}}\left(\Delta, \widetilde{G}_{0}\right)$ and $\operatorname{HF}_{*+1}^{b, b^{\prime}}\left(\Delta, \widetilde{G}_{0}\right)$ can be nontrivial is if they are generated by a clean intersection of type $\widetilde{d}_{0}^{k}$ with $k \geq 2$. In fact, only one of the intervals $\left(a, a^{\prime}\right)$ and $\left(b, b^{\prime}\right)$ can include the action of some $\widetilde{d}_{0}^{k}$. So, by Theorem 4.1 we either have

or

$$
\begin{gathered}
\operatorname{HF}_{*}^{a, a^{\prime}}\left(\Delta, \widetilde{G}_{0}\right)=H_{*-\mu_{\text {Maslov }}\left(\widetilde{d}_{0}^{k}, \widetilde{G}_{0}\right)}\left(S^{2(m+n)-1} ; \mathbb{Z}_{2}\right) \\
\operatorname{HF}_{*+1}^{b, b^{\prime}}\left(\Delta, \widetilde{G}_{0}\right)=H_{*+1-\mu_{\text {Maslov }}\left(\widetilde{d}_{0}^{k}, \widetilde{G}\right)}\left(S^{2(m+n)-1} ; \mathbb{Z}_{2}\right) .
\end{gathered}
$$

Now, the relative index of each $\widetilde{d}_{0}^{k}$ with $k \geq 2$ satisfies

$$
\mu_{\text {Maslov }}\left(\widetilde{d}_{0}^{k}, \widetilde{G}_{0}\right) \geq 3 n_{0}-3 \geq 2 n_{0} .
$$

Thus, for $*=n_{0}$ and $n_{0}-1$, these groups are indeed trivial as required. 
The fact that $H F_{n_{0}}^{a, b}\left(G_{-}\right)=\operatorname{HF}_{n_{0}}^{a, b}\left(\Delta, \widetilde{G}_{-}\right)=\mathbb{Z}_{2}$, can be established using a similar argument. The only difference in this case is that the groups $\operatorname{HF}_{*}^{a, a^{\prime}}\left(\Delta, \widetilde{G}_{-}\right)$ and $\operatorname{HF}_{*+1}^{b, b^{\prime}}\left(\Delta, \widetilde{G}_{-}\right)$may both be nontrivial. However they are still generated by clean intersections of type $\widetilde{d}_{-}^{k}$ for $k \geq 2$, and so for $*=n_{0}$ and $n_{0}-1$ these groups still vanish.

To prove that the map $\sigma_{G_{-} G_{0}}$ is an isomorphism in degree $n_{0}$ we consider the homomorphism induced by the monotone homotopy shown in Figure 3 This homotopy is transversal to the interval $(a, b)$ as per Definition 4.9, and the Morse-Bott nondegenerate submanifolds of periodic orbits, $N_{i}$, are spheres of dimension $2(m+n)-1$.

For simplicity, the total monotone homotopy is broken into two steps. This allows us to factor the total Floer continuation homomorphism $\sigma_{G_{-} G_{0}}$ as the composition of two homomorphisms. We prove that each of these factors is an isomorphism in degree $n_{0}$.

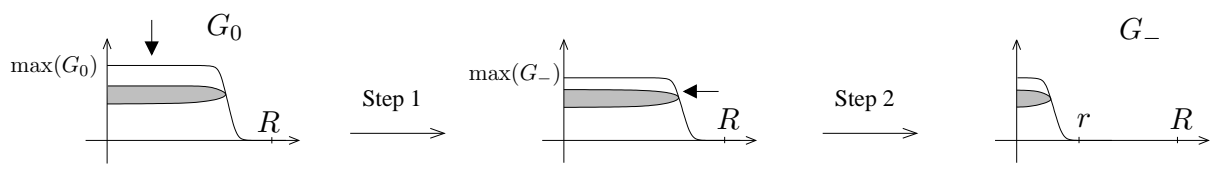

Figure 3: The homotopy in two steps

During the first homotopy, the maximum of the function $G_{0}$ is decreased to coincide with $\max \left(G_{-}\right)$. This is achieved by decreasing the absolute value of the constant slope. Since $\max \left(G_{0}\right)$ and $\max \left(G_{-}\right)$are arbitrarily close, it is clear that the level sets of 1 -periodic orbits change only slightly during this homotopy and no new periodic orbits with period one and action equal to $a$ or $b$ are created. By Lemma 4.8 the homomorphism induced by this step of the homotopy is an isomorphism in Hamiltonian Floer homology restricted to actions in $(a, b)$.

During the second homotopy the radius of the "spike" is decreased but its slope remains constant (see Figure 3). More precisely, the Hamiltonians which occur in this homotopy are of the form $G_{s}=\alpha_{s}\left(\|p\|_{2 m}^{2}+\|z\|^{2}\right)$, and the only change which occurs is that the size of the interval on which $\alpha_{s}$ take its maximum value decreases. Hence, each of the Hamiltonians $G_{s}$ has its 1 -periodic orbits located on two finite sequences of 1-periodic level sets, $\left\{c^{k}(s)\right\}$ and $\left\{d^{k}(s)\right\}$. The relative indices of the these level sets are

$$
\mu_{\mathrm{CZ}}\left(c^{k}(s), G_{s}\right)=(2 k-1)(m+n)+1
$$


and

$$
\mu_{\mathrm{CZ}}\left(d^{k}(s), G_{s}\right)=(2 k-1)(m+n) .
$$

We note that for $k \geq 2$ all of the relative indices are greater than $n_{0}+1=$ $m+n+2$.

The actions $\mathcal{A}_{G_{s}}\left(c^{k}(s)\right)$ and $\mathcal{A}_{G_{s}}\left(d^{k}(s)\right)$ are smooth decreasing functions of $s$. We now determine which of the levels can attain the action values $a$ or $b$. The functions $\mathcal{A}_{G_{s}}\left(c^{k}(s)\right)$ are always greater than $a$ and can only attain the value $b$ when $k \geq 2$, since, for $k=1$, the action starts at $\mathcal{A}_{G_{-1}}\left(c^{1}(-1)\right) \approx \mathcal{A}_{G_{0}}\left(c_{0}^{1}\right)=$ $\max \left(G_{+}\right)+\pi R^{2}+\cdots<b$. The functions $\mathcal{A}_{G_{s}}\left(d^{k}(s)\right)$ can only take the values $a$ or $b$ for $k \geq 2$ since, for $k=1$, the action starts at $\mathcal{A}_{G_{-1}}\left(d^{1}(-1)\right) \approx \mathcal{A}_{G_{0}}\left(d_{0}^{1}\right)=$ $\pi R^{2}+\cdots<a$. As noted above, the relative indices for $k \geq 2$ are all greater than $m+n+2$, so it follows from Proposition 4.10 that the monotone Floer continuation map induced by the second monotone homotopy is an isomorphism in degree $n_{0}=m+n+1$. This completes the proof of Proposition 5.2.

\subsection{Proof of Proposition 5.3}

For $\star=0$ and + , we know that the set $\operatorname{Crit}^{a, b}\left(\widetilde{\mathcal{A}}_{\widetilde{G}_{\star}}\right)$ consists of $\widetilde{c}_{\star}^{1}$ and possibly one other clean intersection of type $\widetilde{d}_{\star}^{k}$ with $k \geq 2$. In considering the map $\sigma_{\widetilde{G}_{0}^{W} \widetilde{G}_{+}^{W}}$ acting on $\operatorname{HF}_{n_{0}}^{a, b}\left(\delta, \widetilde{G}_{+}\right)$, the clean intersections of type $\widetilde{d}_{\star}^{k}$ may again be avoided as they were in the proof of Proposition 5.2. More precisely, we may assume that the actions of $\widetilde{c}_{\star}^{1}$ are distinct from the actions of the $\widetilde{d}_{\star}^{k}$ for $k \geq 2$, and choose constants $a^{\prime}$ and $b^{\prime}$ with $a<a^{\prime}<\widetilde{\mathcal{A}}_{\widetilde{G}_{\star}}\left(\widetilde{c}_{\star}^{1}\right)<b^{\prime}<b$, such that

$$
\operatorname{Crit}^{a^{\prime}, b^{\prime}}\left(\widetilde{\mathcal{A}}_{\widetilde{G}_{\star}^{W}}\right)=\widetilde{c}_{\star}^{1} .
$$

The same arguments used to prove Proposition 5.2 now imply that the groups $\operatorname{HF}_{*}^{a, a^{\prime}}\left(\Delta, \widetilde{G}_{\star}\right)$ and $\operatorname{HF}_{*+1}^{b, b^{\prime}}\left(\Delta, \widetilde{G}_{\star}\right)$ are trivial for $*=n_{0}$ and $*=n_{0}-1$. It then follows from repeated use of Lemma 4.6 and Lemma 4.7 that proving Proposition 5.3 it equivalent to showing that the map

$$
\sigma_{\widetilde{G}_{0}^{W} \widetilde{G}_{+}^{W}}: \operatorname{HF}_{n_{0}}^{a^{\prime}, b^{\prime}}\left(\Delta, \widetilde{G}_{+}^{W}\right) \rightarrow \operatorname{HF}_{n_{0}}^{a^{\prime}, b^{\prime}}\left(\Delta, \widetilde{G}_{0}^{W}\right)
$$

is surjective. To prove this, we will need to study the map $\sigma_{\tilde{G}_{0}^{W}} \widetilde{G}_{+}^{W}$ at the chain level. As described in Remark 4.3. this requires us to consider approximations $\widetilde{G}_{+}^{W^{\prime}}$ of $\widetilde{G}_{+}^{W}$ and $\widetilde{G}_{0}^{W^{\prime}}$ of $\widetilde{G}_{0}^{W}$, such that the 1 -periodic orbits of $\widetilde{G}_{+}^{W^{\prime}}$ and $\widetilde{G}_{0}^{W^{\prime}}$ with actions in $\left(a^{\prime}, b^{\prime}\right)$ are nondegenerate.

Proposition 5.4 The approximations $\widetilde{G}_{+}^{W^{\prime}}$ and $\widetilde{G}_{0}^{W^{\prime}}$ can be constructed so that there is a point $\widetilde{x}_{*}$ in $\widetilde{W}$ with the following properties: 
(1) The point $\widetilde{x}_{*}$ lies in the intersection $\mathrm{Crit}_{n_{0}}^{a^{\prime}, b^{\prime}}\left(\widetilde{\mathcal{A}}_{\widetilde{G}_{+}^{W^{\prime}}}\right) \cap \mathrm{Crit}_{n_{0}}^{a^{\prime}, b^{\prime}}\left(\widetilde{\mathcal{A}}_{\widetilde{G}_{0}^{W^{\prime}}}\right)$.

(2) The class $\left[\widetilde{x}_{*}+\widetilde{w}\right]$ is nontrivial in $\operatorname{HF}_{n_{0}}^{a^{\prime}, b^{\prime}}\left(\Delta, \widetilde{G}_{+}^{W^{\prime}}\right)$ for some chain $\widetilde{w}$ in $\mathrm{CF}_{n_{0}}^{a^{\prime}, b^{\prime}}\left(\Delta, \widetilde{G}_{+}^{W^{\prime}}\right)$ with $\mathcal{A}_{\widetilde{G}_{+}^{W^{\prime}}}(\widetilde{w})<\mathcal{A}_{\widetilde{G}_{+}^{W^{\prime}}}\left(\widetilde{x}_{*}\right)$.

(3) The class $\left[\widetilde{x}_{*}\right]$ is nontrivial and generates $\operatorname{HF}_{n}^{a^{\prime}, b^{\prime}}\left(\Delta, \widetilde{G}_{0}^{W^{\prime}}\right)$.

(4) At the chain level, $\sigma_{\widetilde{G}_{0}^{W^{\prime}}} \widetilde{G}_{+}^{W^{\prime}}\left(\widetilde{x}_{*}+\widetilde{w}\right)=\widetilde{x}_{*}$.

At the level of homology, Proposition 5.4 implies that

$$
\sigma_{\widetilde{G}_{0}^{W^{\prime}} \widetilde{G}_{+}^{W^{\prime}}}\left(\left[\widetilde{x}_{*}+\widetilde{w}\right]\right)=\left[\widetilde{x}_{*}\right] \neq 0 .
$$

In particular, the map

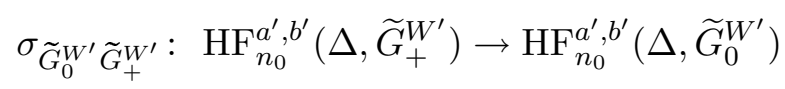

is surjective. By Remark 4.3 the same conclusion holds for $\sigma_{\widetilde{G}_{0}^{W}} \widetilde{G}_{+}^{W}$, and by the discussion above this implies Proposition 5.3 .

\subsection{Proof of Proposition 5.4}

For our choice of $a^{\prime}$ and $b^{\prime}$, we have

$$
\widetilde{c}_{+}^{1}=\operatorname{Crit}^{a^{\prime}, b^{\prime}}\left(\widetilde{\mathcal{A}}_{\widetilde{G}_{+}^{W}}\right) \quad \text { and } \quad \widetilde{c}_{0}^{1}=\operatorname{Crit}^{a^{\prime}, b^{\prime}}\left(\widetilde{\mathcal{A}}_{\widetilde{G}_{0}^{W}}\right) .
$$

Let $h_{+}: \widetilde{c}_{+}^{1} \rightarrow \mathbb{R}$ be a Morse function and let $g^{+}$be a metric on $\widetilde{c}_{+}^{1}$ such that the corresponding Morse complex is well-defined. Similarly choose a Morse function and metric $\left(h_{0}, g_{0}\right)$ on $\widetilde{c}_{0}^{1}$. Theorem 4.1 then yields, for sufficiently small $\delta>0$, the following strong equivalences of chain complexes:

$$
\left(C\left(h_{+}\right), \partial_{g_{+}}\right) \equiv\left(\mathrm{CF}^{a^{\prime}, b^{\prime}}\left(\Delta, \widetilde{G}_{+}^{W}+\delta \widetilde{H}_{h_{+}}\right), \partial_{J_{g_{+}, t}}\right)
$$

and

$$
\left(C\left(h_{0}\right), \partial_{g_{0}}\right) \equiv\left(\mathrm{CF}^{a^{\prime}, b^{\prime}}\left(\Delta, \widetilde{G}_{0}^{W}+\delta \widetilde{H}_{h_{0}}\right), \partial_{J_{g_{0}, t}}\right) .
$$

We recall here the important point that this strong equivalence implies that the critical points of the Morse functions are exactly the points of intersection which generate the corresponding Lagrangian Floer complexes. The equivalences (18) and (19) will be used to prove Proposition 5.4 at the level of Morse homology. In particular, we will set $\widetilde{G}_{+}^{W^{\prime}}=\widetilde{G}_{+}^{W}+\delta \widetilde{H}_{h_{+}}$and $\widetilde{G}_{0}^{W^{\prime}}=\widetilde{G}_{0}^{W}+\delta \widetilde{H}_{h_{0}}$ for special choices of the Morse functions $h_{+}$and $h_{0}$.

The definition of these functions involves the following simple model. Let

$$
\text { height }_{k}: S^{k} \subset \mathbb{R}^{k+1} \rightarrow \mathbb{R}
$$


denote the height function on the standard $k$-dimensional unit sphere given by the restriction of the function

$$
\left(x_{1}, \ldots, x_{k+1}\right) \mapsto x_{1} .
$$

For a (linear) subsphere of $S^{k}$ defined by $l$ linear conditions of the form $x_{j}=0$ where $j \neq 1$, the restriction of height ${ }_{k}$ clearly agrees with the function height ${ }_{k-l}$ and the critical points of the two functions coincide.

Recall that $\widetilde{c}_{+}^{1}$ is an $S^{2 n-1}$-bundle over $M$. To define $h_{+}$we first choose a Morse function $f_{M}: M \rightarrow \mathbb{R}$ which, for simplicity, we assume is self-indexing and has only one critical point of index $2 m$ at $p_{\text {fix }} \in M$. In other words, if $\operatorname{Crit}\left(f_{M}\right)=\left\{p_{j}\right\}$, then

$$
f_{M}\left(p_{j}\right)=\mu_{\text {Morse }}\left(p_{j}, h_{+}\right) \text {, and }\left(f_{M}\right)^{-1}(2 m)=p_{2 m}=p_{\text {fix }} .
$$

The lift of $f_{M}$ to $\widetilde{c}_{+}^{1}$ is a Morse-Bott function whose critical submanifolds correspond to the fibres of $\widetilde{c}_{+}^{1}$ over the points $p_{j}$. To define $h_{+}$we perturb the lift of $f_{M}$ near each of its critical fibres as follows

$$
h_{+}(p, z)=f_{M}(p)+\epsilon \sum_{p_{j} \in \operatorname{Crit}\left(f_{M}\right)} \eta_{j}(p) \text { height }_{2 n-1}(z) .
$$

Here, each $\eta_{j}$ is a smooth bump function on $M$ which has support near $p_{j}$ and attains its maximum value, one, in a neighborhood of $p_{j}$. For sufficiently small $\epsilon>0, h_{+}$is a Morse function whose critical points lie in the fibres over the points $p_{j} \in \operatorname{Crit}\left(f_{M}\right)$ where they coincide with the critical points of the function height ${ }_{2 n-1}$ on the fibre. In other words, each critical point $p_{j}$ of $f_{M}$ gives rise to exactly two critical points of the perturbation $h_{+}$, which we denote by $x_{j,+}^{\mathrm{top}}$ and $x_{j,+}^{\mathrm{bottom}}$. The Morse index of $x_{j}^{\mathrm{top}}$ is given by

$$
\mu_{\text {Morse }}\left(x_{j,+}^{\text {top }}\right)=\mu_{\text {Morse }}\left(p_{j}\right)+2 n-1
$$

and similarly

$$
\mu_{\text {Morse }}\left(x_{j,+}^{\mathrm{bottom}}\right)=\mu_{\mathrm{Morse}}\left(p_{j}\right) .
$$

Note, that the function $h_{+}$restricts to the fibre of $\widetilde{c}_{+}^{1}$ over $p_{j}$ as

$$
f_{M}\left(p_{j}\right)+\epsilon \cdot \text { height }_{2 n-1} \text {. }
$$

Now, the level set $\widetilde{c}_{0}^{1}$ is diffeomorphic to $S^{2(m+n)-1}$, and, by the definitions of $G_{+}$and $G_{0}$, it is clear that $\widetilde{c}_{+}^{1} \cap \widetilde{c}_{0}^{1}$ is equal to the fibre of $\widetilde{c}_{+}^{1}$ over $p_{\text {fix }} \in M$. We define the Morse function $h_{0}: \widetilde{c}_{0}^{1} \approx S^{2(m+n)-1} \rightarrow \mathbb{R}$ by the equation

$$
h_{0}=f_{M}\left(p_{\text {fix }}\right)+\epsilon \cdot \text { height }_{2(m+n)-1} .
$$

As described above, the restriction of $h_{0}$ to $\widetilde{c}_{+}^{1} \cap \widetilde{c}_{0}^{1}$ agrees with the restriction of $h_{+}$(see (20) ) and the two critical points of $h_{0}$, which we call $x_{0}^{\text {top }}$ and $x_{0}^{\text {bottom }}$, coincide with $x_{2 m,+}^{\text {top }}$ and $x_{2 m,+}^{\text {bottom }}$, respectively. 


\subsubsection{Proof of part (1)}

Set

$$
\widetilde{x}_{*}=x_{2 m,+}^{\text {bottom }}=x_{0}^{\text {bottom }} .
$$

Under the identifications (18) and (19), it follows that the point $\widetilde{x}_{*} \in \widetilde{W}$ belongs to both $\operatorname{Crit}^{a^{\prime}, b^{\prime}}\left(\widetilde{\mathcal{A}}_{\widetilde{G}_{+}^{W^{\prime}}}\right)$ and $\mathrm{Crit}^{a^{\prime}, b^{\prime}}\left(\widetilde{\mathcal{A}}_{\widetilde{G}_{0}^{W^{\prime}}}\right)$.

The critical point $x_{2 m++}^{\text {bottom }}$ has Morse index $2 m$ and the clean intersection $\widetilde{c}_{+}^{1}$ has relative Maslov index $n-m+1$. It follows from Theorem 4.1 that $\widetilde{x}_{*}$ has Maslov index $(2 m)+(n-m+1)=m+n+1$ as an element of $\mathrm{Crit}^{a^{\prime}, b^{\prime}}\left(\widetilde{\mathcal{A}}_{\widetilde{G}_{+}^{W^{\prime}}}\right)$. Similarly, $\widetilde{x}_{*}$ has Maslov index $m+n+1$ as an element of $\mathrm{Crit}^{a^{\prime}, b^{\prime}}\left(\widetilde{\mathcal{A}}_{\widetilde{G}_{0}^{W^{\prime}}}\right)$.

\subsubsection{Proof of part (2)}

Let $g_{+}$be a metric on $\widetilde{c}_{+}^{1}$ for which the Morse chain complex $\left(C\left(h_{+}\right), \partial_{g_{+}}\right)$is well-defined. This complex has a natural filtration coming from the function $f_{M}$ on $M$. More precisely, let $C_{i, j}\left(h_{+}\right)$be the $\mathbb{Z}_{2}$-vector space generated by the critical points $x$ of $h_{+}$such that $\mu_{\text {Morse }}\left(x, h_{+}\right)=i+j$ and $x$ lies in the fibre over some $p_{k} \in \operatorname{Crit}\left(f_{M}\right)$ where $\mu_{\text {Morse }}\left(p_{k}, f_{M}\right)=i$. Then

$$
C_{k}=\bigoplus_{i+j=k} C_{i, j}
$$

admits the filtration

$$
\mathcal{F}_{0} C_{k} \subseteq \mathcal{F}_{1} C_{k} \cdots \subseteq \mathcal{F}_{k} C_{k}
$$

where

$$
\mathcal{F}_{j} C_{k}=\bigoplus_{i \leq j} C_{i, k-i}
$$

Since we are only considering coefficients in the field $\mathbb{Z}_{2}$, the corresponding spectral sequence converges to $H_{*}\left(\widetilde{c}_{+}^{1}, \mathbb{Z}_{2}\right)$. It is easy to see that the $E^{1}$-term is given by

$$
E_{i, j}^{1}=C_{i}\left(f_{M}\right) \otimes H_{j}\left(S^{2 n-1}, \mathbb{Z}_{2}\right)=C_{i, j}
$$

Lemma 5.5 The $E^{2}$-term of the spectral sequence is

$$
E_{i, j}^{2}=H_{i}\left(M, H_{j}\left(S^{2 n-1}, \mathbb{Z}_{2}\right)\right) .
$$

A proof of this well-known result has been included in Appendix B. 
To prove Part (2) of Proposition 5.4 we first note that $E_{2 m, 0}^{1}=\mathbb{Z}_{2}$ and is represented by $\widetilde{x}_{*}=x_{2 m,+}^{\text {bottom }}$. Let $\beta_{k}$ denote the $k^{\text {th }} \mathbb{Z}_{2}$-Betti number of $M$. It follows from Lemma 5.5 that

$$
E_{2 m}^{2}=E_{2 m, 0}^{2} \oplus E_{2(m-n)+1,2 n-1}^{2}=\mathbb{Z}_{2} \oplus \mathbb{Z}_{2}^{\beta_{2(m-n)+1}} .
$$

In addition, the assumption that the unit normal bundle of $M$ is homologically trivial in degree $2 m$ implies that

$$
H_{2 m}\left(\widetilde{c}_{+}^{1}, \mathbb{Z}_{2}\right)=\mathbb{Z}_{2} \oplus \mathbb{Z}_{2}^{\beta_{2(m-n)+1}}
$$

Since the spectral sequence converges to $H_{*}\left(\widetilde{c}_{+}^{1}, \mathbb{Z}_{2}\right)$, equations (21) and (22) imply that $E_{2 m, 0}^{k}$ is isomorphic to $\mathbb{Z}_{2}$ and is generated by $\left[\widetilde{x}_{*}\right]$ for all $k \geq 0$. Moreover, $\widetilde{x}_{*}$ must appear nontrivially in a representative of a nonzero class in $H_{2 m}\left(\widetilde{c}_{+}^{1}, \mathbb{Z}_{2}\right)$. We denote this class by $\left[\widetilde{x}_{*}+\widetilde{w}\right]$

By the strong equivalence of complexes from (18) and our choice of $h^{+}$it is clear that $\widetilde{x}_{*}$ has the largest action in $\mathrm{Crit}_{n_{0}}^{a^{\prime}, b^{\prime}}\left(\widetilde{\mathcal{A}}_{\widetilde{G}_{+}^{W^{\prime}}}\right)$. This completes the proof of Part (2).

\subsubsection{Proof of part (3)}

The point $\widetilde{x}_{*}=x_{0}^{\text {bottom }}$ is the unique global minimum of $h_{0}$. Since

$$
H_{*}\left(C\left(h_{0}\right), \partial_{g_{0}}\right)=H_{*}\left(\widetilde{c}_{0}^{1}, \mathbb{Z}_{2}\right)=H_{*}\left(S^{2(m+n)-1}, \mathbb{Z}_{2}\right),
$$

the class $\left[\widetilde{x}_{*}\right]$ must represent the nontrivial class $H_{0}\left(C\left(h_{0}\right), \partial_{g_{0}}\right)$. Part $(3)$ then follows immediately from the strong equivalence of complexes from (19).

\subsubsection{Proof of part (4)}

Let $\widetilde{v}_{+} \in \operatorname{Crit}^{a^{\prime}, b^{\prime}}\left(\widetilde{G}_{+}^{W^{\prime}}\right)$ and $\widetilde{v}_{0} \in \operatorname{Crit}^{a^{\prime}, b^{\prime}}\left(\widetilde{G}_{0}^{W^{\prime}}\right)$. For a monotone homotopy $\widetilde{G}_{s}^{W^{\prime}}$ from $\widetilde{G}_{+}^{W^{\prime}}$ to $\widetilde{G}_{0}^{W^{\prime}}$ and a generic family of almost complex structures $J_{s, t}$, consider an element $\widetilde{u} \in \widetilde{\mathcal{M}}_{s}\left(\widetilde{v}_{+}, \widetilde{v}_{0}, \widetilde{G}_{s}^{W^{\prime}}, \widetilde{J}_{s, t}\right)$. By inequality (12), we have

$$
\widetilde{\mathcal{A}}_{\widetilde{G}_{0}^{W^{\prime}}}\left(\widetilde{v}_{0}\right) \leq \widetilde{\mathcal{A}}_{\widetilde{G}_{+}^{W^{\prime}}}\left(\widetilde{v}_{+}\right)
$$

with equality only when $\widetilde{u}(s, t)$ does not depend on $s$.

Setting $\widetilde{v}_{+}=\widetilde{x}_{*}=\widetilde{v}_{0}$ and noting that $\widetilde{\mathcal{A}}_{\widetilde{G}_{+}^{W^{\prime}}}\left(\widetilde{x}_{*}\right)=\widetilde{\mathcal{A}}_{\widetilde{G}_{0}^{W^{\prime}}}\left(\widetilde{x}_{*}\right)$ it follows that the moduli space $\widetilde{\mathcal{M}}_{s}\left(\widetilde{x}_{*}, \widetilde{x}_{*}, \widetilde{G}_{s}^{W^{\prime}}, \widetilde{J}_{s, t}\right)$ contains only the constant map.

We also know that $\widetilde{x}_{*}$ has the smallest $\left(\widetilde{\mathcal{A}}_{\widetilde{G}_{0}^{W^{\prime}}}\right)$-value in $\mathrm{Crit}^{a^{\prime}, b^{\prime}}\left(\widetilde{\mathcal{A}}_{\widetilde{G}_{0}^{W^{\prime}}}\right)$. Since any $\widetilde{v}_{+}$which appears in $\widetilde{w}$ with nonzero coefficient satisfies

$$
\widetilde{\mathcal{A}}_{\widetilde{G}_{+}^{W^{\prime}}}\left(\widetilde{v}_{+}\right)<\widetilde{\mathcal{A}}_{\widetilde{G}_{0}^{W^{\prime}}}\left(\widetilde{x}_{*}\right)
$$


we have $\widetilde{\mathcal{M}}_{s}\left(\widetilde{v}_{+}, \widetilde{x}_{*}, \widetilde{G}_{s}^{W^{\prime}}, \widetilde{J}_{s, t}\right)=\emptyset$.

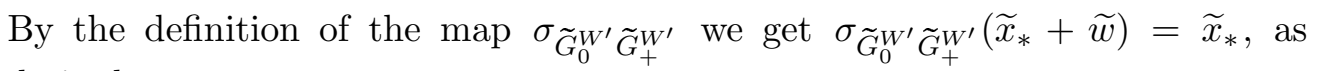
desired.

\section{Appendices}

\section{A Area bounds for plane curves with positive cur- vature}

We begin by recalling the statement of Proposition 2.12

Proposition A.1 Let $\xi:[0, T] \rightarrow \mathbb{R}^{2}$ be a closed planar curve with constant speed $v$, rotation number $k$, and positive curvature $K(t) / v$ such that $0<\underline{K} \leq K(t)$. The Euclidean area enclosed by $\xi, A(\xi)$, satisfies

$$
0 \leq A(\xi) \leq k 4(v / \underline{K})^{2} .
$$

Proof A closed piecewise linear curve is called a box curve if each segment is either vertical or horizontal. We can assume that as one traverses the curve the segments alternate between vertical and horizontal. A box curve is said to be positive if one always makes a left turn between segments as one follows the curve in a counterclockwise manner. Note that any positive box curve is completely determined (up to translation) by two finite sequences of positive numbers $\left\{a_{j}\right\}_{j=1}^{2 k}$ and $\left\{b_{j}\right\}_{j=1}^{2 k}$ such that

$$
\sum_{j=1}^{2 k}(-1)^{j+1} a_{j}=0=\sum_{j=1}^{2 k}(-1)^{j+1} b_{j} .
$$

Here the $a_{j}$ are the lengths of the vertical segments and the $b_{j}$ are the lengths of the horizontal segments and we use the convention that the first segment corresponds to $a_{1}$. The rotation number of the curve is clearly equal to $k$. (See Figure 4)

To prove Proposition 2.12 we will associate to $\xi(t)$ a positive box curve $\widehat{\xi}(t)$ such that

$$
A(\xi) \leq A(\widehat{\xi}) \leq k 4(v / \underline{K})^{2} .
$$

Note that

$$
\dot{\xi}(t)=(v \cos \theta(t), v \sin \theta(t))
$$

where $\theta(t)$ is the solution of the initial value problem

$$
\dot{\theta}=K(t), \quad \theta(0)=0 .
$$




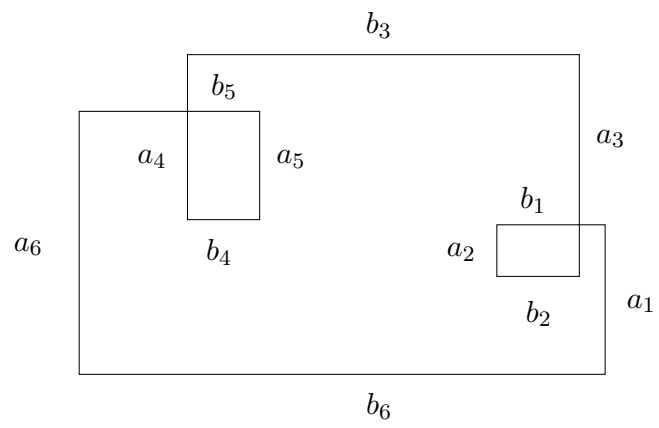

Figure 4: A positive box curve

We define the value $t_{j}$ as the unique solution of the equation

$$
\theta\left(t_{j}\right)=j \pi
$$

Similarly, we let $\tau_{j}$ be the solution of

$$
\theta\left(\tau_{j}\right)=\frac{\pi}{2}+j \pi
$$

For $j=1, \ldots, 2 k$ we then set

$$
a_{j}=y\left(t_{j}\right)-y\left(t_{j-1}\right) \quad \text { and } \quad b_{j}=x\left(t_{j}\right)-x\left(t_{j-1}\right) .
$$

These sequences determine the box curve $\widehat{\xi}$ illustrated in Figure 5 Clearly,

$$
A(\widehat{\xi}) \geq A(\xi) \text {. }
$$

Moreover, for all $j=1, \ldots, 2 k$ we have

$$
a_{j}, b_{j} \leq 2 v / \underline{K}
$$

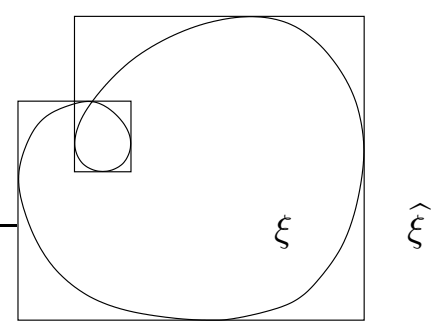

Figure 5: The curves $\xi$ and $\widehat{\xi}$ 
Lemma A.2 Let $\widehat{\xi}$ be a positive box curve determined by the sequences $\left\{a_{j}\right\}_{j=1}^{2 k}$ and $\left\{b_{j}\right\}_{j=1}^{2 k}$. Suppose that

$$
a_{j}, b_{j} \leq \bar{c}
$$

for all $j=1, \ldots, 2 k$. Then

$$
0<A(\widehat{\xi}) \leq k \bar{c}^{2}
$$

Proof For convenience we set

$$
\tilde{a}_{i}=\sum_{j=1}^{i}(-1)^{j+1} a_{j}
$$

The area of $\widehat{\xi}$ is then given by the formula

$$
A(\widehat{\xi})=b_{1} \widetilde{a}_{1}-b_{2} \widetilde{a}_{2}+\ldots+b_{2 k-1} \widetilde{a}_{2 k-1} .
$$

Consider the positive box curve $\bar{\xi}$ determined by the sequences $\left\{\bar{a}_{j}\right\}_{j=1}^{2 k}$ and $\left\{\bar{b}_{j}\right\}_{j=1}^{2 k}$ where $\bar{a}_{j}=a_{j}$ and $\bar{b}_{j}=\bar{c}$. We then have

$$
\begin{aligned}
A(\bar{\xi}) & =\bar{c} \widetilde{a}_{1}-\bar{c} \widetilde{a}_{2}+\ldots+\bar{c} \widetilde{a}_{2 k-1} \\
& =\left(b_{1} \widetilde{a}_{1}-\ldots+b_{2 k-1} \widetilde{a}_{2 k-1}\right)+\left(\left(c-b_{1}\right) \widetilde{a}_{1}-\ldots+\left(c-b_{2 k-1}\right) \widetilde{a}_{2 k-1}\right) \\
& =A(\widehat{\xi})+\left(\left(c-b_{1}\right) \widetilde{a}_{1}-\ldots+\left(c-b_{2 k-1}\right) \widetilde{a}_{2 k-1}\right) .
\end{aligned}
$$

The second term is the area of the positive box corresponding to the sequences $\left\{a_{j}\right\}_{j=1}^{2 k}$ and $\left\{\bar{c}-b_{j}\right\}_{j=1}^{2 k}$. Hence this term is nonnegative and we have

$$
A(\bar{\xi}) \geq A(\widehat{\xi}) .
$$

Finally,

$$
A(\bar{\xi})=\bar{c}\left(\widetilde{a}_{1}-\widetilde{a}_{2}+\cdots+\widetilde{a}_{2 k-1}\right)=\bar{c}\left(a_{1}+a_{3}+\cdots+a_{2 k-1}\right) \leq k \bar{c}^{2} .
$$

Applying Lemma A.2 to the positive box curve $\widehat{\xi}$ defined by $\xi$, we obtain Proposition A.1.

\section{B Spectral sequences for the Morse homology of fi- bre bundles}

Let $p r: P \rightarrow B$ be a fibre bundle with a closed base $B$ and with closed fibres diffeomorphic to a manifold $F$. Let $h_{B}: B \rightarrow \mathbb{R}$ be a self-indexing Morse function with critical points $\left\{b_{j}\right\}$, ie $h_{B}\left(b_{j}\right)=\mu_{\text {Morse }}\left(b_{j}, h_{B}\right)$. The lift of $h_{B}$ via $p r$ is a Morse-Bott function on $P$ whose critical submanifolds are the fibres of $P$ over the points $b_{j}$. We can construct from $h_{B}$ a useful Morse function on $P$ by perturbing $p r^{*} h_{B}$ close to 
its critical fibres as follows. Let $\mathcal{V}_{j}$ be a small open neighborhood of $b_{j}$ in $B$ over which the bundle $P$ is trivial and let $\eta_{j}$ be a smooth bump function on $B$ which is supported in $\mathcal{V}_{j}$ and attains its maximum in a neighborhood of $b_{j}$. Define the function $h: P \rightarrow \mathbb{R}$ by

$$
h(b, z)=h_{B}(b)+\epsilon \sum_{b_{j} \in \operatorname{Crit}\left(h_{B}\right)} \eta_{j}(b) f(z),
$$

where $f: F \rightarrow \mathbb{R}$ is chosen to be Morse. For sufficiently small $\epsilon>0, h$ is Morse and all its critical points lie in the fibres $p r^{-1}\left(b_{j}\right) \approx F$ where they coincide with the critical points of the function $f$.

Fix a metric $g$ on $P$ for which the Morse chain complex $\left(C(h), \partial_{g}\right)$ is well-defined. That is, we assume that the stable and unstable manifolds of the gradient vector field

$$
V_{h, g}=\operatorname{grad}(h, g)
$$

intersect transversally. The chain group $C(h)$ is the $\mathbb{Z}_{2}$-vector space generated by the critical points of $h$. The boundary operator is defined using the moduli spaces, $m(x, y)$, of integral curves of $-V_{h, g}(x)$ going from $x \in \operatorname{Crit}(h)$ to $y \in \operatorname{Crit}(h)$. By our assumption on $V_{h, g}$, each $\mathrm{m}(x, y)$ is a smooth manifold of dimension $\mu_{\text {Morse }}(x, h)-$ $\mu_{\text {Morse }}(y, h)$ on which $\mathbb{R}$ acts freely by translation. The map $\partial_{g}$ is then defined by

$$
\partial_{g}(x)=\sum_{\mu_{\text {Morse }}(y, h)=\mu_{\text {Morse }}(x, h)-1} \#(m(x, y) / \mathbb{R}) \cdot y,
$$

where $\#(m(x, y) / \mathbb{R})$ is the number of elements in $m(x, y) / \mathbb{R}$ modulo 2 .

Our special choice of the function $h$ yields a natural filtration for the chain complex $\left(C(h), \partial_{g}\right)$. More precisely, let $C_{i, j}(h)$ be the $\mathbb{Z}_{2}$-vector space generated by the critical points $x$ of $h$ that satisfy

$$
\mu_{\text {Morse }}(x, h)=i+j \quad \text { and } \quad \mu_{\text {Morse }}\left(\operatorname{pr}(x), h_{B}\right)=i .
$$

Then each graded component

$$
C_{k}(h)=\bigoplus_{i+j=k} C_{i, j}(h)
$$

admits the filtration

$$
\mathcal{F}_{0} C_{k}(h) \subseteq \mathcal{F}_{1} C_{k}(h) \cdots \subseteq \mathcal{F}_{k} C_{k}(h),
$$

where

$$
\mathcal{F}_{j} C_{k}(h)=\bigoplus_{i \leq j} C_{i, k-i}(h) .
$$

The assumption that $h_{B}$ is self-indexing implies the following useful fact: For $x \in$ $C_{i, j}(h)$ and $y \in C_{i^{\prime}, j^{\prime}}(h)$ the moduli space $m(x, y)$ is empty whenever $i^{\prime}>i$. This follows from the fact that $h(x)<h(y)$. It implies that the boundary map respects the filtration and we get a spectral sequence $E_{i, j}^{k}$. 
Let $\partial^{k}: C_{i, j}(h) \rightarrow C_{i-k, j+k-1}(h)$ be the map which is defined on the generators $x \in$ $C_{i, j}(h)$ by

$$
\partial^{k}(x)=\sum_{\substack{\mu_{\text {Morse }}(y, h)=i+j-1, \mu_{\text {Morse }}\left(\operatorname{pr}(y), h_{B}\right)=i-k}} \#(m(x, y) / \mathbb{R}) \cdot y .
$$

The Morse boundary operator $\partial_{g}$ decomposes as

$$
\partial_{g}=\sum_{k \geq 0} \partial^{k}
$$

Moreover, for each $k \geq 0$ we have $\partial^{k} \circ \partial^{k}=0$ and

$$
E_{i, j}^{k+1}=H_{*}\left(E_{i, j}^{k}, \partial^{k}\right) .
$$

Since we are using coefficients in the field $\mathbb{Z}_{2}$ the spectral sequence converges to $H_{*}\left(P, \mathbb{Z}_{2}\right)$. That is, $E_{i, j}^{k}=E_{i, j}^{k+1}=E_{i, j}^{\infty}$ for all sufficiently large $k$, and

$$
H_{l}\left(P, \mathbb{Z}_{2}\right)=\bigoplus_{i+j=l} E_{i, j}^{\infty}
$$

It is easy to see that the $E^{1}$-terms are given by

$$
E_{i, j}^{1}=C_{i}\left(h_{B}\right) \otimes H_{j}\left(F, \mathbb{Z}_{2}\right) .
$$

The point of this appendix is to provide a proof of the following well-known result.

Proposition B.1 The $E^{2}$-term of the spectral sequence is

$$
E_{i, j}^{2}=H_{i}\left(B, H_{j}\left(F, \mathbb{Z}_{2}\right)\right)
$$

Our proof of Proposition B.1 utilizes the generalization of Morse homology to gradientlike vector fields. This argument is motivated by the recent work of Hutchings [40].

Recall that a vector field $V$ is a gradient-like vector field for a Morse function $h: N^{k} \rightarrow$ $\mathbb{R}$ if

- $d h(V)>0$ away from the critical points of $h$,

- near each critical point $x$ of $h, V$ has the form of a negative gradient vector field $-V_{h, g}$ for some metric $g$.

If $V$ is gradient-like for $h$ and the stable and unstable manifolds of its flow intersect transversely, then $V$ is said to be Morse-Smale. Given such a vector field, one can construct a Morse complex $\left(C(h), \partial_{V}\right)$ in the usual way.

The resulting homology $H_{*}\left(C(h), \partial_{V}\right)$ does not depend on the choice of $V$ and is equal to $H_{*}(N)$. To see this consider two Morse-Smale gradient-like vector fields for $h, V_{1}$ 
and $V_{2}$. For $s \in \mathbb{R}$ let $V_{s}$ be a family of vector fields such that $V_{s}=V_{1}$ for all $s \leq-1$, $V_{s}=V_{2}$ for all $s \geq 1$, and $d h\left(V_{s}\right)>0$ away from Crit $(h)$ for all $s \in \mathbb{R}$ (such families always exist). As in Floer theory, this determines a continuation chain map

$$
\sigma_{V_{2}, V_{1}}:\left(C(h), \partial_{V_{1}}\right) \rightarrow\left(C(h), \partial_{V_{2}}\right)
$$

The map is defined by counting the solutions $u: \mathbb{R} \rightarrow N$ of the equation

$$
\dot{u}(s)=-V_{s}(u(s)) \text {. }
$$

The usual arguments imply that at the homology level $\sigma_{V_{3}, V_{2}} \circ \sigma_{V_{2}, V_{1}}=\sigma_{V_{3}, V_{1}}$ and $\sigma_{V, V}=I d$. Hence $H_{*}\left(C(h), \partial_{V}\right)$ does not depend on $V$. Letting $V=\operatorname{grad}(h, g)$ for a generic metric $g$ on $N$ it follows that

$$
H_{*}\left(C(h), \partial_{V}\right)=H_{*}(N) \text {. }
$$

\section{B.1 Proof of Proposition B.1}

Let $V_{1}=V_{h, g}$. The idea of the proof of Proposition B.1 is to first construct another gradient-like vector field $V_{2}$ for $h$ which admits its own spectral sequence $\mathcal{E}_{i, j}^{k}$ such that $\mathcal{E}_{i, j}^{2}=H_{i}\left(B, H_{j}\left(F, \mathbb{Z}_{2}\right)\right)$. Then we show that the continuation map $\sigma_{V_{1}, V_{2}}$ induces an isomorphism from $E_{i, j}^{2}$ to $\mathcal{E}_{i, j}^{2}$.

To define $V_{2}$ we follow [40. Let $h_{B}: B \rightarrow \mathbb{R}$ and $f: F \rightarrow \mathbb{R}$ be the functions used to define $h$. Let $g_{B}$ be a metric on $B$ for which the gradient vector field $V_{B}=$ $\operatorname{grad}\left(h_{B}, g_{B}\right)$ is Morse-Smale. Let $g_{F}$ be a fibrewise metric on the total space $P$ and let $V_{F}$ be the fibrewise gradient field of $f$ with respect to $g_{F}$. Fixing a connection on $P$ we set

$$
V_{2}=V_{F}+\operatorname{Hor}\left(V_{B}\right),
$$

where $\operatorname{Hor}\left(V_{B}\right)$ is the horizontal lift of $V_{B}$. Clearly, $V_{2}$ is gradient-like for $h$. For a generic choice of the fibrewise metric $g_{F}, V_{2}$ is also Morse-Smale 40. In addition, we may assume that the pairs $\left(f, g_{F}\left(b_{j}\right)\right)$ are Morse-Smale for every critical point $b_{j}$ of $h_{B}$.

The boundary map of the corresponding Morse complex $\left(C(h), \partial_{V_{2}}\right)$ also respects the filtration

$$
\mathcal{F}_{0} C_{k}(h) \subseteq \mathcal{F}_{1} C_{k}(h) \cdots \subseteq \mathcal{F}_{k} C_{k}(h),
$$

and yields a second spectral sequence $\mathcal{E}_{i, j}^{k}$.

Lemma B.2 $\mathcal{E}_{i, j}^{2}=H_{i}\left(B, H_{j}\left(F, \mathbb{Z}_{2}\right)\right)$

Proof The following identification is obvious

$$
\mathcal{E}_{i, j}^{0}=C_{i, j}(h)=C_{i}\left(h_{B}\right) \otimes C_{j}(f) .
$$


The boundary operator $\partial_{V}$ decomposes as

$$
\partial_{V}=\sum_{k \geq 0} \partial_{V}^{k}
$$

where each $\partial^{k}: C_{i, j}(h) \rightarrow C_{i-k, j+k-1}(h)$ is defined as above. Hence,

$$
\mathcal{E}_{i, j}^{k+1}=H_{*}\left(\mathcal{E}_{i, j}^{k}, \partial^{k}\right),
$$

and we have

$$
\mathcal{E}_{i, j}^{1}=C_{i}\left(h_{B}\right) \otimes H_{j}\left(F, \mathbb{Z}_{2}\right) .
$$

It suffices for us to prove that

$$
\partial_{V}^{1}(b \otimes[\alpha])=\partial_{g_{B}}(b) \otimes[\alpha],
$$

for each critical point $b \in \operatorname{Crit}_{i}\left(h_{B}\right)$ and each class $[\alpha] \in H_{j}\left(F, \mathbb{Z}_{2}\right)$.

By definition

$$
\partial_{V}^{1}(b \otimes[\alpha])=\sum_{c \in \operatorname{Crit}_{i-1}\left(h_{B}\right)} \sum_{[\beta] \in H_{j}\left(F, \mathbb{Z}_{2}\right)} \#(m(b \otimes \alpha, c \otimes \beta) / \mathbb{R}) c \otimes[\beta] .
$$

Consider a trajectory $\gamma \in m(b \otimes \alpha, c \otimes \beta)$. By the definition of $V_{2}$ the projection of $\gamma$ to $B$ is a gradient trajectory $\gamma_{B}$ of $h_{B}$ with respect to $g_{B}$. Let us choose a trivialization of $P$ over $\gamma_{B}$ and note that this yields a homotopy of functions $h_{s}=\left.h\right|_{p r^{-1}\left(\gamma_{B}(s)\right)}$ on $F$ and metrics $g_{s}=g_{F}\left(\gamma_{B}(s)\right)$ on $F$.

In our trivialization the set of trajectories of $V_{2}$ which project to $\gamma_{B}$ are then distinguished by their fibre components. It is easy to see that these fibre components are exactly the trajectories counted in the Morse continuation map from $\left(C(f), \partial_{g_{F}(b)}\right)$ to $\left(C(f), \partial_{g_{F}(c)}\right)$ that is determined by the homotopies $h_{s}$ and $g_{s}$. Since the continuation map induces an isomorphism in homology we have $[\alpha]=[\beta]$.

Summing over the gradient trajectories of $h_{B}$ from $b$ to $c$ we obtain (23).

Lemma B.3 The map $\sigma_{V_{1}, V_{2}}$ is a morphism of filtered complexes and hence it induces an isomorphism from $E_{i, j}^{k}$ to $\mathcal{E}_{i, j}^{k}$ for all $k \geq 0$.

Proof By construction $\sigma_{V_{1}, V_{2}}$ is a chain map. To prove that it is a morphism of filtered complexes it remains to show that $\sigma_{V_{1}, V_{2}}\left(\mathcal{F}_{i} C_{*}(h)\right) \subset \mathcal{F}_{i} C_{*}(h)$. Let $x \in \mathcal{F}_{i} C_{*}(h)$ and suppose that $y$ appears in the image of $x$ under $\sigma_{V_{1}, V_{2}}$. It follows from the definition of the map $\sigma_{V_{1}, V_{2}}$ that $h(y) \leq h(x)$. Since $h$ is a perturbation of the self-indexing function $h_{B}$ this implies that $y \in \mathcal{F}_{i} C_{*}(h)$. 
Squeezing in Floer theory and refined HZ-capacities

1831

\section{References}

[1] V I Arnol'd, On some problems in symplectic topology, from: "Topology and geometry - Rohlin Seminar", Lecture Notes in Math. 1346, Springer, Berlin (1988) 1-5 MathReview

[2] V Benci, H Hofer, P H Rabinowitz, A remark on a priori bounds and existence for periodic solutions of Hamiltonian systems, from: "Periodic solutions of Hamiltonian systems and related topics (Il Ciocco, 1986)", NATO Adv. Sci. Inst. Ser. C Math. Phys. Sci. 209, Reidel, Dordrecht (1987) 85-88 MathReview

[3] V Benci, PH Rabinowitz, A priori bounds for periodic solutions of a class of Hamiltonian systems, Ergodic Theory Dynam. Systems 8 (1988) 2731 MathReview

[4] M Bialy, L Polterovich, Geodesics of Hofer's metric on the group of Hamiltonian diffeomorphisms, Duke Math. J. 76 (1994) 273-292 MathReview

[5] P Biran, Lagrangian barriers and symplectic embeddings, Geom. Funct. Anal. 11 (2001) 407-464 MathReview

[6] P Biran, L Polterovich, D Salamon, Propagation in Hamiltonian dynamics and relative symplectic homology, Duke Math. J. 119 (2003) 65-118 MathReview

[7] S V Bolotin, Libration motions of natural dynamical systems, Vestnik Moskov. Univ. Ser. I Mat. Mekh. 3 (1978) 72-77 MathReview

[8] $\mathbf{R}$ Bott, L W Tu, Differential forms in algebraic topology, Graduate Texts in Mathematics 82, Springer-Verlag, New York (1982) MathReview

[9] G Contreras, L Macarini, G P Paternain, Periodic orbits for exact magnetic flows on surfaces, Int. Math. Res. Notices 8 (2004) 361-387 MathReview

[10] K Cieliebak, A Floer, H Hofer, Symplectic homology II: A general construction, Math. Z. 218 (1995) 103-122 MathReview

[11] K Cieliebak, A Floer, H Hofer, K Wysocki, Applications of symplectic homology. II. Stability of the action spectrum, Math. Z. 223 (1996) 27-45 MathReview

[12] K Cieliebak, V L Ginzburg, E Kerman, Symplectic homology and periodic orbits near symplectic submanifolds, Comment. Math. Helv. 79 (2004) 554-581 MathReview

[13] C C Conley, E Zehnder, The Birkhoff-Lewis fixed point theorem and a conjecture of VI Arnol'd, Invent. Math. 73 (1983) 33-49 MathReview

[14] Y Eliashberg, A Givental, H Hofer, Introduction to symplectic field theory, Geom. Funct. Anal. (2000) 560-673 MathReview

[15] A Floer, Morse theory for Lagrangian intersections, J. Differential Geom. 28 (1988) 513-547 MathReview 
[16] A Floer, Witten's complex and infinite dimensional Morse Theory, J. Diff. Geom. 30 (1989) 202-221

[17] A Floer, Symplectic fixed points and holomorphic spheres, Comm. Math. Phys. 120 (1989) 575-611 MathReview

[18] A Floer, H Hofer, Symplectic homology I: Open sets in $\mathbb{C}^{n}$, Math. Z. 215 (1994) 37-88 MathReview

[19] A Floer, H Hofer, K Wysocki, Applications of symplectic homology I, Math. Z. 217 (1994) 577-606 MathReview

[20] A Floer, H Hofer, C Viterbo, The Weinstein conjecture on $P \times \mathbb{C}^{l}$, Math. Z. 203 (1989) 355-378

[21] V L Ginzburg, New generalizations of Poincaré's geometric theorem, Funct. Anal. Appl. 21 (1987) 100-106

[22] V L Ginzburg, On closed trajectories of a charge in a magnetic field. An application of symplectic geometry, from: "Contact and symplectic geometry (Cambridge, 1994)", Publ. Newton Inst. 8, Cambridge Univ. Press, Cambridge (1996) 131-148 MathReview

[23] V L Ginzburg, An embedding $S^{2 n-1} \rightarrow \mathbb{R}^{2 n}, 2 n-1 \geq 7$, whose Hamiltonian flow has no periodic trajectories, Internat. Math. Res. Notices 2 (1995) 83-97 MathReview

[24] V L Ginzburg, A smooth counterexample to the Hamiltonian Seifert conjecture in $\mathbb{R}^{6}$, Internat. Math. Res. Notices 13 (1997) 641-650 MathReview

[25] V L Ginzburg, B Z Gürel, $A C^{2}$-smooth counterexample to the Hamiltonian Seifert conjecture in $\mathbb{R}^{4}$, Ann. of Math. (2) 158 (2003) 953-976 MathReview

[26] V L Ginzburg, B Z Gürel, Relative Hofer-Zehnder capacity and periodic orbits in twisted cotangent bundles, Duke Math. J. 123 (2004) 1-47 MathReview

[27] VL Ginzburg, B Z Gürel, E Kerman, Branching Floer Homology, in progress

[28] V L Ginzburg, E Kerman, Periodic orbits in magnetic fields in dimensions greater than two, from: "Geometry and topology in dynamics (Winston-Salem, NC, 1998/San Antonio, TX, 1999)", Contemp. Math. 246, Amer. Math. Soc. Providence, RI (1999) 113-121 MathReview

[29] V L Ginzburg, E Kerman, Periodic orbits of Hamiltonian flows near symplectic extrema, Pacific J. Math. 206 (2002) 69-91 MathReview

[30] A Gray, Tubes, Addison-Wesley Publishing Company Advanced Book Program, Redwood City, CA (1990) MathReview

[31] M-R Herman, Examples of compact hypersurfaces in $\mathbb{R}^{2 p}, 2 p \geq 6$, with no periodic orbits, from: "Hamiltonian systems with three or more degrees of freedom", NATO Adv. Sci. Inst. Ser. C Math. Phys. Sci. 533, Kluwer Acad. Publ. Dordrecht (1999) 
Squeezing in Floer theory and refined HZ-capacities

[32] H Hofer, On the topological properties of symplectic maps, Proc. Roy. Soc. Edinburgh Sect. A 115 (1990) 25-38 MathReview

[33] H Hofer, Estimates for the energy of a symplectic map, Comment. Math. Helv. 68 (1993) 48-72 MathReview

[34] H Hofer, D A Salamon, Floer homology and Novikov rings, from: "The Floer memorial volume", Progr. Math. 133, Birkhäuser, Basel (1995) 483-524 MathReview

[35] H Hofer, C Viterbo, The Weinstein conjecture in cotangent bundles and related results, Ann. Scuola Norm. Sup. Pisa Cl. Sci. (4) 15 (1988) 411-445 (1989) MathReview

[36] H Hofer, C Viterbo, The Weinstein conjecture in the presence of holomorphic spheres, Comm. Pure Appl. Math. 45 (1992) 583-622 MathReview

[37] H Hofer, E Zehnder, Periodic solutions on hypersurfaces and a result by $C$. Viterbo, Invent. Math. 90 (1987) 1-9 MathReview

[38] H Hofer, E Zehnder, A new capacity for symplectic manifolds, from: "Analysis, et cetera", Academic Press, Boston, MA (1990) 405-427 MathReview

[39] H Hofer, E Zehnder, Symplectic invariants and Hamiltonian dynamics, Birkhäuser Advanced Texts, Birkhäuser Verlag, Basel (1994) MathReview

[40] M Hutching, Floer homology of families I, preprint (2003)

[41] E Kerman, Periodic orbits of Hamiltonian flows near symplectic critical submanifolds, Internat. Math. Res. Notices 17 (1999) 953-969 MathReview

[42] E Kerman, New smooth counterexamples to the Hamiltonian Seifert conjecture, J. Symplectic Geom. 1 (2002) 253-267 MathReview

[43] E Kerman, F Lalonde, Length minimizing Hamiltonian paths for symplectically aspherical manifolds, Ann. Inst. Fourier 53 (2003) 1503-1526 MathReview

[44] F Lalonde, D McDuff, Hofer's $L^{\infty}$-geometry: energy and stability of Hamiltonian flows: I, II, Invent. Math. 122 (1995) 1-33, 35-69 MathReview

[45] F Laudenbach, Homotopie régulière inactive et engouffrement symplectique, Ann. Inst. Fourier (Grenoble) 36 (1986) 93-111 MathReview

[46] M Levi, On a problem by Arnold on periodic motions in magnetic fields, Comm. Pure Appl. Math. 56 (2003) 1165-1177 MathReview

[47] G Liu, G Tian, Weinstein conjecture and $G W$-invariants, Commun. Contemp. Math. 2 (2000) 405-459 MathReview

[48] G Lu, The Weinstein conjecture on some symplectic manifolds containing the holomorphic spheres, Kyushu J. Math. 52 (1998) 331-351 MathReview

[49] G Lu, Gromov-Witten invariants and pseudo symplectic capacities, to appear in Israel J. Math. 
[50] L Macarini, Hofer-Zehnder capacity and Hamiltonian circle actions, Commun. Contemp. Math. 6 (2004) 913-945 MathReview

[51] L Macarini, F Schlenk, A refinement of the Hofer-Zehnder theorem on the existence of closed characteristics near a hypersurface, Bull. London Math. Soc. 37 (2005) 297-300 MathReview

[52] D McDuff, Geometric variants of the Hofer norm, J. Symplectic Geom. 1 (2002) 197-252 MathReview

[53] D McDuff, J Slimowitz, Hofer-Zehnder capacity and length minimizing Hamiltonian paths, Geom. Topol. 5 (2001) 799-830 MathReview

[54] Y-G Oh, Chain level Floer theory and Hofer's geometry of the Hamiltonian diffeomorphism group, Asian J. Math. 6 (2002) 579-624 MathReview

[55] Y Ostrover, A comparison of Hofer's metrics on Hamiltonian diffeomorphisms and Lagrangian submanifolds, Commun. Contemp. Math. 5 (2003) 803-811 MathReview

[56] L Polterovich, Geometry on the group of Hamiltonian diffeomorphisms, from: "Proceedings of the International Congress of Mathematicians, Vol II (Berlin 1998)", Doc. Math. (1998) 401-410 MathReview

[57] L Polterovich, The geometry of the group of symplectic diffeomorphisms, Lectures in Mathematics ETH Zürich, Birkhäuser Verlag, Basel (2001) MathReview

[58] M Poźniak, Floer homology, Novikov rings and clean intersections, from: "Northern California Symplectic Geometry Seminar", Amer. Math. Soc. Transl. Ser. 2 196, Amer. Math. Soc. Providence, RI (1999) 119-181 MathReview

[59] F Schlenk, Applications of Hofer's geometry to Hamiltonian dynamics, to appear in Comment. Math. Helvetici.

[60] M Schwarz, On the action spectrum for closed symplectically aspherical manifolds, Pacific J. Math. 193 (2000) 419-461 MathReview

[61] D Salamon, Lectures on Floer homology, from: "Symplectic geometry and topology (Park City, UT, 1997)", IAS/Park City Math. Ser. 7, Amer. Math. Soc. Providence, RI (1999) 143-229 MathReview

[62] K F Siburg, New minimal geodesics in the group of symplectic diffeomorphisms, Calc. Var. Partial Differential Equations 3 (1995) 299-309 MathReview

[63] J-C Sikorav, Systèmes Hamiltoniens et topologie symplectique, ETS, EDITRICE Pisa (1990)

[64] M Struwe, Existence of periodic solutions of Hamiltonian systems on almost every energy surface, Bol. Soc. Brasil. Mat. (N.S.) 20 (1990) 49-58 MathReview

[65] I Ustilovsky, Conjugate points on geodesics of Hofer's metric, Differential Geom. Appl. 6 (1996) 327-342 MathReview

[66] H Weyl, On the Volume of Tubes, Amer. J. Math. 61 (1939) 461-472 MathReview 